\title{
STABLE SET-VALUED INTEGRATION OF NONLINEAR DYNAMIC SYSTEMS USING AFFINE SET-PARAMETERIZATIONS*
}

\author{
BORIS HOUSKA ${ }^{\dagger}$, MARIO E. VILLANUEVA ${ }^{\ddagger}$, AND BENOÎT CHACHUAT ${ }^{\S}$
}

\begin{abstract}
Many set-valued integration algorithms for parametric ordinary differential equations (ODEs) implement a combination of Taylor series expansion with either interval arithmetic or Taylor model arithmetic. Due to the wrapping effect, the diameter of the solution-set enclosures computed with these algorithms typically diverges to infinity on finite integration horizons, even though the ODE trajectories themselves may be asymptotically stable. This paper starts by describing a new discretized set-valued integration algorithm that uses a predictor-validation approach to propagate generic affine set-parameterizations, whose images are guaranteed to enclose the ODE solution set. Sufficient conditions are then derived for this algorithm to be locally asymptotically stable, in the sense that the computed enclosures are guaranteed to remain stable on infinite time horizons when applied to a dynamic system in the neighborhood of a locally asymptotically stable periodic orbit (or equilibrium point). The key requirement here is quadratic Hausdorff convergence of function extensions in the chosen affine set-parameterization, which is proved to be the case, for instance, for Taylor models with ellipsoidal remainders. These stability properties are illustrated with the case study of a cubic oscillator system.
\end{abstract}

Key words. ordinary differential equations, reachability analysis, affine set-parameterization, set-valued integration, convergence analysis, stability analysis

AMS subject classifications. 34D05, 34D35, 65G40, 65L05, 65L70, 90-08, 90C26, 93D20

DOI. $10.1137 / 140976807$

1. Introduction. Enclosing the reachable set of nonlinear ordinary differential equations (ODEs), also known as validated ODE integration or set-valued ODE integration, finds applications in many research fields, including reachability analysis for control systems, robust optimal control, and global optimization of dynamic systems. In the field of reachability analysis $[4,30]$, bounds on parametric ODEs can be used for assessing the stability or the controllability of a given linear or nonlinear process. This generic bounding capability is also pivotal in deterministic global optimization of dynamic systems in order to bracket the optimal solution value and in turn provide a certificate of global optimality $[6,15,19,29]$, as well as in robust optimal control as a means to enforce constraint satisfaction $[13,14,17]$.

Existing methods for set-valued ODE integration can be broadly classified into either continuous or discretized approaches. In the first class, auxiliary differential equations are formulated, whose solutions describe an enclosure of the original ODE

* Received by the editors July 9, 2014; accepted for publication (in revised form) August 3, 2015; published electronically October 20, 2015. The research of the authors was supported by the Engineering and Physical Sciences Research Council (EPSRC) grant EP/J006572/1, National Science Foundation China (NSFC) grant 61473185 as well as ShanghaiTech University grant F-0203-14-012, Marie Curie Career Integration grant PCIG09-GA-2011-293953, and the Centre of Process Systems Engineering (CPSE) of Imperial College.

http://www.siam.org/journals/sinum/53-5/97680.html

${ }^{\dagger}$ Centre for Process Systems Engineering, Department of Chemical Engineering, Imperial College London, London SW7 2AZ, UK and School of Information Science and Technology, ShanghaiTech University, Shanghai, 200031, China (borish@shanghaitech.edu.ch).

${ }^{\ddagger}$ Centre for Process Systems Engineering, Department of Chemical Engineering, Imperial College London, London SW7 2AZ, UK (mario.villanueva10@imperial.ac.uk). The research of the second author was supported by a CONACYT doctoral scholarship.

$\S$ Corresponding author. Centre for Process Systems Engineering, Department of Chemical Engineering, Imperial College London, London SW7 2AZ, UK (b.chachuat@imperial.ac.uk). 
solutions pointwise in time. The classical theory of differential inequalities [39] provides a means for propagating an enclosure of the reachable set in the form of an interval vector. This approach has been later extended to propagate affine bounds [36], a pair of convex/concave bounds [33, 34], Taylor models [7], and ellipsoidal bounds [14]. Recently, a unified framework based on a generalized differential inequality has also been presented, which allows analyzing the convergence properties of various continuous-time set-valued integration approaches [38]. Nonetheless, developing a fully validated, yet practical, integrator based on this approach presents several challenges, mainly due to the need for applying a numerical discretization to solve the auxiliary ODEs. Because the right-hand side function of the auxiliary bounding ODE is typically nondifferentiable, no guarantee on the discretization error can be given in general when classical integration schemes such as Runge-Kutta methods [10] are used. This nondifferentiability can also impair the step-size control mechanism of the numerical integration algorithm, and as such it should be treated as events in the framework of hybrid discrete-continuous systems; see, for instance, [35].

In contrast, discretized set-valued integration proceeds by first discretizing the integration horizon into finite steps and it can account for discretization errors in propagating the solution enclosures through each step. Many such validated integrators go back to the original work by Moore [23], who presented a simple test for checking the existence and uniqueness of ODE solutions over a finite time step using interval analysis. This test was later incorporated into an algorithm that discretizes the integration horizon into finite steps and proceeds in two phases [20, 8, 26]: (i) determine a step-size and an a priori enclosure of the ODE solutions over the current step then (ii) propagate a tightened enclosure until the end of that step. In particular, the second phase relies on a high-order Taylor expansion of the ODE solutions in time, for instance, evaluated using interval arithmetic or Taylor model arithmetic with interval remainder bounds $[3,18,27]$. The propagation of convex/concave bounds, using either McCormick relaxations [21] or McCormick-Taylor models [5], has also been proposed [31, 32].

Both continuous and discretized set-valued integration methods are subject to the wrapping effect, which typically results in the diameter of the reachable set enclosure diverging to infinity, even on finite time horizons - the so-called bound explosion phenomenon. For stable ODE systems in particular, a rather natural requirement would appear to be that the computed enclosures are themselves stable, at least for small enough initial value or uncertain parameter sets. Nonetheless, most existing validated ODE integrators fail to satisfy this property. Moreover, the question under which conditions the resulting enclosures are stable or convergent has seldom been investigated so far. In order to avoid confusion at this point, we note that these stability issues are of different nature than those arising in the context of stiff ODEs, which have been analyzed extensively for both standard (nonvalidated) integration algorithms [10] as well as for validated integrators based on implicit integration schemes [26]. Because of the wrapping effect, the computed enclosure of the reachable set can indeed become unstable no matter how small the steps taken by the integrator are. Therefore, the focus here is on explicit integration schemes, while extensions to handle stiff dynamic systems are only mentioned briefly toward the end of the paper.

In this context, the main contribution of this paper is two-fold:

(i) We propose a novel discretized set-valued ODE integration algorithm, which reverses the classical two-phase approach of validated ODE integration by first constructing a predictor of the reachable set and then determining a step-size for which this predictor yields a valid enclosure. This reversed approach leads to a natural 
step-size control mechanism, which no longer relies on the availability of an a priori enclosure. Moreover, we formulate the algorithm generically in terms of affine setparameterizations, thus encompassing the propagation of interval boxes, ellipsoids, and Taylor models.

(ii) We analyze the stability of the reachable set enclosures obtained with the developed algorithm. Our main contribution here is to prove that, for a certain class of (asymptotically) stable parametric ODEs, locally stable and convergent enclosures can be obtained on infinite time horizons when the underlying affine set arithmetic exhibits quadratic Hausdorff convergence.

The rest of the paper is organized as follows. Both the problem formulation and the notation used throughout the paper are specified in the following subsections. Section 2 introduces the class of affine set-parameterizations for enclosing the range of vector functions and discusses convergence considerations. The discretized set-valued ODE integration algorithm is presented in section 3 , and stability properties of the computed enclosures are analyzed in section 4 . These properties are illustrated with a numerical case study in section 5. Finally, section 6 concludes the paper.

1.1. Problem statement. We consider parametric dynamic systems in the form of nonlinear ODEs

$$
\forall t \in[0, T], \quad \dot{x}(t, p)=f(t, x(t, p), p) \quad \text { with } \quad x(0, p)=x_{0}(p) .
$$

Assumptions on the right-hand side function $f: \mathbb{R} \times \mathbb{R}^{n_{x}} \times \mathbb{R}^{n_{p}} \rightarrow \mathbb{R}^{n_{x}}$ and the initial value function $x_{0}: \mathbb{R}^{n_{p}} \rightarrow \mathbb{R}^{n_{x}}$ will be specified later on in the paper. The state $x:[0, T] \times P \rightarrow \mathbb{R}^{n_{x}}$ is regarded as a function of the uncertain parameter vector $p \in P \subseteq \mathbb{R}^{n_{p}}$ along the time horizon $[0, T]$. The reachable set of the initial value problem (1.1) is denoted by

$$
X(t, P):=\{x(t, p) \mid p \in P\}
$$

or simply $X(t)$ when it is clear from the context what the corresponding parameter host set $P$ is.

The focus here is on algorithms that compute a time-varying enclosure $Y(t, P) \supseteq$ $X(t, P)$ for all $t \in[0, T]$ using discretized set-valued integration techniques. Particular emphasis is on analyzing whether, as well as determining under which conditions, one can obtain stable enclosures $Y(t, P)$ on infinite time horizons for sufficiently small (yet finite) parameter host sets $P$.

Originally introduced by McCormick [21] for the development of a convex/concave relaxation arithmetic, factorable functions cover an extremely inclusive class of functions which can be represented finitely on a computer by means of a code list or a computational graph involving atom operations. These are typically unary and binary operations within a library of atom operators, which can be based for example on the C-code library math.h. Besides convex/concave relaxations, factorable functions find applications in automatic differentiation (AD) $[9,25]$ as well as in interval analysis [24] and Taylor model arithmetic [28].

A major complication with the solutions $x(t, \cdot)$ of the parametric ODEs (1.1) is that these functions do not have a factorable representation in general, and therefore classical bounding techniques based on interval analysis or Taylor model arithmetic cannot be applied directly. Nonetheless, algorithms for bounding the solution set of parametric ODEs take advantage of the fact that the right-hand side function $f$ and the initial value function $x_{0}$ are typically factorable. 
1.2. Notation. Besides standard mathematical notation, the diameter of a compact set $Z \subseteq \mathbb{R}^{n}$ is defined as

$$
\operatorname{diam}(Z):=\max _{z, z^{\prime} \in Z}\left\|z-z^{\prime}\right\|
$$

for any given norm on $\mathbb{R}^{n}$. The Minkowski sum $W \oplus Z$ and the Hausdorff distance $d_{\mathrm{H}}(W, Z)$ between two compact sets $W, Z \in \mathbb{R}^{n}$ are given by

$$
\begin{aligned}
W \oplus Z & :=\{w+z \mid w \in W, z \in Z\}, \\
d_{\mathrm{H}}(W, Z) & :=\max \left\{\max _{w \in W} \min _{z \in Z}\|w-z\|, \max _{z \in Z} \min _{w \in W}\|w-z\|\right\} .
\end{aligned}
$$

If $W \subseteq Z$ in particular, we have

$$
d_{\mathrm{H}}(W, Z)=\max _{z \in Z} \min _{w \in W}\|w-z\| .
$$

Moreover, by a small abuse of notation, we denote the Hausdorff distance between a compact set $Z \subseteq \mathbb{R}^{n}$ and the origin by

$$
\|Z\|_{\mathrm{H}}:=d_{\mathrm{H}}(Z,\{0\})=\max _{z \in Z}\|z\|,
$$

although the function $\|\cdot\|_{\mathrm{H}}$ does not define a norm in general.

2. Bounding of factorable vector-valued functions using affine set-parameterizations. This section introduces a framework based on affine set-parameterizations for bounding the image-set of factorable, vector-valued functions. Considerations on Hausdorff convergence of these function extensions are discussed, and a technique based on Taylor model arithmetic that constructs quadratically Hausdorff convergent extensions is presented.

2.1. Affine set-parameterizations. We start by defining the class of computerrepresentable sets of interest.

Definition 2.1. Let $\mathbb{E}_{\ell} \subseteq \mathbb{R}^{\ell}$ and $\mathbb{D}_{n, \ell} \subseteq \mathbb{R}^{n \times(\ell+1)}$ with $\ell \geq 1$ and $n \geq 1$. For any $Q \in \mathbb{D}_{n, \ell}$, we define the image of $\mathbb{E}_{\ell}$ under the affine map $\xi \mapsto Q\left(\xi^{\mathrm{T}}, 1\right)^{\mathrm{T}}$ as

$$
\operatorname{Im}_{\mathbb{E}_{\ell}}(Q):=\left\{Q\left(\xi^{\mathrm{T}}, 1\right)^{\mathrm{T}} \mid \xi \in \mathbb{E}_{\ell}\right\} \subset \mathbb{R}^{n} .
$$

In this set-representation, $Q$ is referred to as the parameterization, and the pair $\left(\mathbb{E}_{\ell}, \mathbb{D}_{n, \ell}\right)$ is called an affine set-parameterization, with $\mathbb{E}_{\ell}$ and $\mathbb{D}_{n, \ell}$ the basis set and the domain set, respectively.

Usual families of convex sets such as intervals, ellipsoids, zonotopes or polytopes can all be represented using affine set-parameterizations with convex basis sets.

Example 1. Every $\ell$-dimensional ellipsoid in $\mathbb{R}^{n}$ can be represented using an affine set-parameterization with the basis set

$$
\mathbb{E}_{\ell}^{\text {ball }}:=\left\{\xi \in \mathbb{R}^{\ell} \mid\|\xi\|_{2} \leq 1\right\}
$$

and the associated domain set $\mathbb{R}^{n \times(\ell+1)}$. Likewise, every polytope and every zonotope in $\mathbb{R}^{n}$ can be represented using affine set-parameterizations with the basis sets

$$
\mathbb{E}_{\ell}^{\text {simplex }}:=\left\{\xi \in \mathbb{R}_{+}^{\ell} \mid\|\xi\|_{1} \leq 1\right\} \quad \text { and } \quad \mathbb{E}_{\ell}^{\text {box }}:=\left\{\xi \in \mathbb{R}^{\ell} \mid\|\xi\|_{\infty} \leq 1\right\},
$$


respectively, and the same domain set $\mathbb{R}^{n \times(\ell+1)}$. The latter basis set $\mathbb{E}_{\ell}^{\text {box }}$ can also be associated with the domain

$$
\mathbb{D}_{\ell}^{\text {interval }}:=\left\{(\operatorname{diag}(r), c) \mid r \in \mathbb{R}_{+}^{\ell}, c \in \mathbb{R}^{\ell}\right\}
$$

in order to describe interval boxes in $\mathbb{R}^{\ell}$ with radius $r$ and centered at $c$.

Affine set-parameterizations can also be used to describe certain classes of nonconvex sets by considering nonconvex basis sets.

Example 2. The affine set-parameterization $\left(\mathbb{E}_{\ell}^{\mathrm{pol}(q)}, \mathbb{R}^{n \times\left(\alpha_{\ell}^{(q)}+1\right)}\right)$ with

$$
\mathbb{E}_{\ell}^{\mathrm{pol}(q)}:=\left\{M_{\ell, q}(\xi) \mid \xi \in[-1,1]^{\ell}\right\},
$$

where $M_{\ell, q}(\xi) \in \mathbb{R}^{\alpha_{\ell}^{(q)}}$ is the vector containing the first $\alpha_{\ell}^{(q)}$ monomials in $\xi$ in lexicographic order,

$$
M_{\ell, q}(\xi):=\left(\xi_{1}, \xi_{2}, \ldots, \xi_{\ell}, \xi_{1}^{2}, \xi_{1} \xi_{2}, \ldots, \xi_{\ell}^{2}, \xi_{1}^{3}, \ldots, \xi_{\ell}^{q}\right)^{\mathrm{T}},
$$

describes nonconvex sets in $\mathbb{R}^{n}$-we shall refer to this parameterization as the class of qth-order polynomial models subsequently. Clearly, other bases than the monomial basis $M_{\ell, q}(\xi)$ can be used in (2.1), such as the Legendre basis or the Chebyshev basis. More generally, any $q$ th-order polynomial model can be combined with a convex set, e.g., in the manner of the remainder term in a Taylor model [28] to account for higherorder terms. Considering interval boxes as the convex set, such a combination leads to the parameterization $\left(\mathbb{E}_{\ell}^{\mathrm{pol}(q)} \times \mathbb{E}_{n}^{\mathrm{box}}, \mathbb{R}^{n \times \alpha_{\ell}^{(q)}} \times \mathbb{D}_{n}^{\text {interval }}\right)$; with ellipsoids, likewise, it leads to the affine set-parameterization $\left(\mathbb{E}_{\ell}^{\text {pol }(q)} \times \mathbb{E}_{n}^{\text {ball }}, \mathbb{R}^{n \times\left(\alpha_{\ell}^{(q)}+n+1\right)}\right)$. In the latter construct, the first $n$-by- $\alpha_{\ell}^{(q)}$ block of the parameter $Q \in \mathbb{R}^{n \times\left(\alpha_{\ell}^{(q)}+n+1\right)}$ comprises the multivariate polynomial coefficients; the following $n$-by- $n$ block, the shape matrix coefficients of the ellipsoid; and the last $n$-by- 1 block, the center of the ellipsoid.

Of special interest for the stability analysis conducted later on in the paper is the concept of invariance under affine transformation, as defined next.

Definition 2.2. An affine set-parameterization $\left(\mathbb{E}_{\ell}, \mathbb{D}_{n, \ell}\right)$ is said to be invariant under affine transformation if for every affine map $x \mapsto A x+b$ with $(A, b) \in \mathbb{R}^{m \times(n+1)}$ and every $Q \in \mathbb{D}_{n, \ell}$,

$$
\exists Q^{\prime} \in \mathbb{D}_{m, \ell}: \quad \operatorname{Im}_{\mathbb{E}_{\ell}}\left(Q^{\prime}\right)=\left\{A x+b \mid x \in \operatorname{Im}_{\mathbb{E}_{\ell}}(Q)\right\}=\operatorname{Im}_{\mathbb{E}_{\ell}}(A Q) \oplus\{b\} .
$$

Remark 1. An immediate consequence of the property of invariance under affine transformation is that the image of $\mathbb{E}_{\ell}$ under the composition of two affine transformations, say, given by the parameterizations $Q \in \mathbb{D}_{n, \ell}$ and $P \in \mathbb{R}^{m \times(n+1)}$, can always be represented exactly as the image of $\mathbb{E}_{\ell}$ under a third affine transformation with $Q^{\prime} \in \mathbb{D}_{m, \ell}$ such that

$$
\operatorname{Im}_{\mathbb{E}_{\ell}}\left(Q^{\prime}\right)=\operatorname{Im}_{\operatorname{Im}_{\mathbb{E}_{\ell}}(Q)}(P)
$$

Among the convex and nonconvex set representations considered in Examples 1 and 2 , some, but not all, are invariant under affine transformation.

Example 3. Given an ellipsoid $\operatorname{Im}_{\mathbb{E}_{\ell}^{\text {ball }}}(Q)$ with $Q \in \mathbb{R}^{n \times(\ell+1)}$, the application of any affine transformation $x \mapsto A x+b$ with $(A, b) \in \mathbb{R}^{m \times(n+1)}$ yields another ellipsoid $\operatorname{Im}_{\mathbb{E}_{\ell}^{\text {ball }}}\left(Q^{\prime}\right)$ with

$$
Q^{\prime}:=A Q+\left(0_{m \times \ell}, b\right) \in \mathbb{R}^{m \times(\ell+1)} .
$$


Therefore, the class of ellipsoids is invariant under affine transformation. The same parameters $Q^{\prime}$ can be used to show that the classes of polytopes, zonotopes, and polynomial models - as well as any finite combination of these parameterizationsare also invariant under affine transformation.

Example 4. The rotation of an interval box in $\mathbb{R}^{n}$ may yield another interval box whose edges are no longer aligned with the original axes, in which case the transformed box cannot be represented exactly in terms of the affine parameterization $\left(\mathbb{E}_{n}^{\text {box }}, \mathbb{D}_{n}^{\text {interval }}\right)$ anymore. In other words, the class of interval boxes is not invariant under affine transformation, which is one of the main sources of the wrapping effect in interval analysis. It also follows that any affine set-parameterizations obtained from the combination with interval boxes, e.g., polynomial models with interval boxes as in Example 2, will fail to be invariant under affine transformation.

2.2. Affine set-parameterization extensions of vector-valued functions. Natural interval extensions and their variants [24] were among the first techniques developed for bounding the range of factorable functions. The concept of interval extension in interval analysis extends readily to affine set-parameterizations.

Definition 2.3. Consider a function $\varphi: \mathbb{R}^{n} \rightarrow \mathbb{R}^{m}$, and let $\left(\mathbb{E}_{1}, \mathbb{D}_{1}\right)$ and $\left(\mathbb{E}_{2}, \mathbb{D}_{2}\right)$ be two affine set-parameterizations. The function $\varphi^{\mathbb{E}_{1}, \mathbb{E}_{2}}: \mathbb{D}_{1} \rightarrow \mathbb{D}_{2}$ is called an extension of $\varphi$ from $\mathbb{D}_{1}$ to $\mathbb{D}_{2}$ if

$$
\forall Q \in \mathbb{D}_{1}, \quad \operatorname{Im}_{\mathbb{E}_{2}}\left(\varphi^{\mathbb{E}_{1}, \mathbb{E}_{2}}(Q)\right) \supseteq \bar{\varphi}^{\mathbb{E}_{1}}(Q),
$$

where $\bar{\varphi}^{\mathbb{E}_{1}}(Q):=\left\{\varphi(x) \mid x \in \operatorname{Im}_{\mathbb{E}_{1}}(Q)\right\}$ denotes the exact image of $\varphi$ on $\operatorname{Im}_{\mathbb{E}_{1}}(Q)$. In the special case that $\mathbb{E}_{1}=\mathbb{E}_{2}=: \mathbb{E}$, we use the shorthand notation $\varphi^{\mathbb{E}}$.

A special notation is also defined for extensions of the binary addition operation for convenience. We shall only use this notation when it is clear from the context what the basis and domain of the affine set-parameterization are.

Definition 2.4. Let $(\mathbb{E}, \mathbb{D})$ be an affine set-parameterization. An extension of the binary addition is a function $\uplus: \mathbb{D} \times \mathbb{D} \rightarrow \mathbb{D}$ such that

$$
\forall Q, Q^{\prime} \in \mathbb{D}, \quad \operatorname{Im}_{\mathbb{E}}\left(Q \uplus Q^{\prime}\right) \supseteq\left\{x+x^{\prime} \mid x \in \operatorname{Im}_{\mathbb{E}}(Q), x^{\prime} \in \operatorname{Im}_{\mathbb{E}}\left(Q^{\prime}\right)\right\} .
$$

Moreover, $\uplus$ is said to be a regular addition extension if

$$
\forall Q, Q^{\prime} \in \mathbb{D}, \quad d_{\mathrm{H}}\left(\operatorname{Im}_{\mathbb{E}}\left(Q \uplus Q^{\prime}\right), \operatorname{Im}_{\mathbb{E}}(Q)\right)=\mathbf{O}\left(\left\|\operatorname{Im}_{\mathbb{E}}\left(Q^{\prime}\right)\right\|_{H}\right) .
$$

Note that (2.2) only imposes a mild regularity condition, which is automatically satisfied when using either interval arithmetic [24] or Taylor model arithmetic [1, 2, 28] and can easily be satisfied with ellipsoidal calculus too.

A key property of the affine set-parameterization extension of a function is how much overestimation it carries with respect to the actual image set of that function. Especially relevant for the stability analysis conducted in this paper is the Hausdorff convergence order.

Definition 2.5. The extension $\varphi^{\mathbb{E}_{1}, \mathbb{E}_{2}}: \mathbb{D}_{1} \rightarrow \mathbb{D}_{2}$ of a function $\varphi: \mathbb{R}^{n} \rightarrow \mathbb{R}^{m}$ is said to have Haussdorf convergence order $q \geq 1$ if

$$
\forall Q \in \mathbb{D}_{1}, \quad d_{\mathrm{H}}\left(\operatorname{Im}_{\mathbb{E}_{2}}\left(\varphi^{\mathbb{E}_{1}, \mathbb{E}_{2}}(Q)\right), \bar{\varphi}^{\mathbb{E}_{1}}(Q)\right)=\mathbf{O}\left(\operatorname{diam}\left(\operatorname{Im}_{\mathbb{E}_{1}}(Q)\right)^{q}\right) .
$$

In the case that an affine set-parameterization is not invariant under affine transformation, constructing extensions that have Hausdorff convergence order two or 
higher is not possible in general. This is even so for extensions of simple linear functions, as illustrated below in the case of interval analysis.

Example 5. Consider the affine set-parameterization $\left(\mathbb{E}_{2}^{\text {box }}, \mathbb{D}_{2}^{\text {interval }}\right)$ and let $Q_{\delta}:=\left(\operatorname{diag}(\delta, \delta),(0,0)^{\mathrm{T}}\right) \in \mathbb{D}_{2}^{\text {interval }}$. Notice that $\operatorname{Im}_{\mathbb{E}_{2}^{\text {box }}}\left(Q_{\delta}\right)$ describes a twodimensional box centered at the origin and of radius $\delta$, the diameter of which is thus in $\mathbf{O}(\delta)$. Now, consider the linear transformation $\varphi: \mathbb{R}^{2} \rightarrow \mathbb{R}^{2}$ such that

$$
\forall x \in \mathbb{R}^{2}, \quad \varphi(x):=\frac{1}{\sqrt{2}}\left(\begin{array}{c}
x_{1}+x_{2} \\
x_{1}-x_{2}
\end{array}\right) .
$$

The exact image of $Q_{\delta}$ under $\varphi$ is given by

$$
\bar{\varphi}^{\mathbb{E}_{2}^{\text {box }}}\left(Q_{\delta}\right):=\{A x \mid x \in[-\delta, \delta] \times[-\delta, \delta]\} \quad \text { with } \quad A=\frac{1}{\sqrt{2}}\left(\begin{array}{cc}
1 & 1 \\
1 & -1
\end{array}\right),
$$

which is a $45^{\circ}$ rotation of $\operatorname{Im}_{\mathbb{E}_{2}^{\text {box }}}\left(Q_{\delta}\right)$ in the plane, around the origin. On the other hand, the natural interval extension $\varphi^{\mathbb{E}^{\text {box }}}$ of $\varphi$ at $Q_{\delta}$ gives

$$
\varphi^{\mathbb{E}_{2}^{\text {box }}}\left(Q_{\delta}\right):=\sqrt{2} \operatorname{Im}_{\mathbb{E}_{2}^{\text {box }}}\left(Q_{\delta}\right)
$$

which is also the interval hull of $\bar{\varphi}^{\mathbb{E}_{2}^{\text {box }}}\left(Q_{\delta}\right)$. Nonetheless, the Hausdorff distance between the sets $\varphi^{\mathbb{E}_{2}^{\text {box }}}\left(Q_{\delta}\right)$ and $\bar{\varphi}^{\mathbb{E}_{2}^{\text {box }}}\left(Q_{\delta}\right)$ is such that

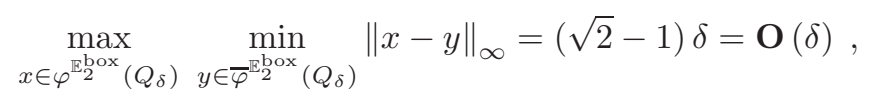

that is, the natural interval extension of $\varphi$ has Hausdorff convergence order 1 .

Remark 2. The affine set-parameterization $\left(\mathbb{E}_{\ell}^{\text {pol }(q)} \times \mathbb{E}_{n}^{\text {box }}, \mathbb{R}^{n \times \alpha_{\ell}^{(q)}} \times \mathbb{D}_{n}^{\text {interval }}\right)$ namely, the class of polynomial models combined with interval boxes - is not invariant under affine transformation for the same reason as in Example 5 before. Therefore, extensions of certain functions may only have Hausdorff convergence order 1 for such parameterization, regardless of the polynomial order $q$. This is the case, for instance, when using Taylor model arithmetic [28] to construct an extension $\varphi^{\mathbb{E}_{\ell}^{\text {pol }(q)} \times \mathbb{E}_{n}^{\text {box }}, \mathbb{E}_{\ell}^{\text {pol }(q)} \times \mathbb{E}_{m}^{\text {box }}}$ from $\mathbb{R}^{m \times \alpha_{\ell}^{(q)}} \times \mathbb{D}_{n}^{\text {interval }}$ to $\mathbb{R}^{m \times \alpha_{\ell}^{(q)}} \times \mathbb{D}_{m}^{\text {interval }}$ of a given factorable and $(q+1)$-times continuously differentiable function $\varphi: \mathbb{R}^{n} \rightarrow \mathbb{R}^{m}$. It is worth mentioning that the foregoing observation is not in contradiction with the convergence analysis in [5], which shows that an extension $\varphi^{\mathbb{E}_{\ell}^{\text {pol }(q)}, \mathbb{E}_{\ell}^{\text {pol }(q)} \times \mathbb{E}_{m}^{\text {box }}}$ from $\mathbb{R}^{n \times \alpha_{\ell}^{(q)}}$ to $\mathbb{R}^{m \times \alpha_{\ell}^{(q)}} \times \mathbb{D}_{m}^{\text {interval }}$ of $\varphi$ in the form of a $q$ th-order Taylor model will have Hausdorff convergence order $q+1$. In the present, more general, context, it is indeed the wrapping of the interval box by the function that causes the loss of convergence order.

The following result is given for future reference in the stability analysis.

Proposition 2.6. Let the extension $\varphi^{\mathbb{E}_{1}, \mathbb{E}_{2}}: \mathbb{D}_{1} \rightarrow \mathbb{D}_{2}$ of a continuouslydifferentiable function $\varphi: \mathbb{R}^{n} \rightarrow \mathbb{R}^{m}$ have quadratic Hausdorff convergence. Then,

$$
\begin{aligned}
d_{\mathrm{H}}\left(\operatorname{Im}_{\mathbb{E}_{2}}\left(\varphi^{\mathbb{E}_{1}, \mathbb{E}_{2}}(Q)\right), \varphi(\xi)+\frac{\partial \varphi}{\partial x}(\xi) \cdot\left[\operatorname{Im}_{\mathbb{E}_{1}}(Q)-\xi\right]\right) \\
=\mathbf{O}\left(\left\|\operatorname{Im}_{\mathbb{E}_{1}}(Q)-\xi\right\|_{\mathrm{H}}^{2}\right)
\end{aligned}
$$

for all $Q \subseteq \mathbb{D}_{1}$ and all $\xi \in \mathbb{R}^{n}$ with $\left\|\operatorname{Im}_{\mathbb{E}_{1}}(Q)-\xi\right\|_{\mathrm{H}}$ sufficiently small. 
Proof. Without loss of generality, we assume $\xi=0$, as we can always shift the sets by a constant offset. The main idea of the proof is to exploit the triangle inequality for the Hausdorff metric, which yields

$$
\begin{aligned}
& d_{\mathrm{H}}\left(\operatorname{Im}_{\mathbb{E}_{2}}\left(\varphi^{\mathbb{E}_{1}, \mathbb{E}_{2}}(Q)\right), \varphi(0)+\frac{\partial \varphi}{\partial x}(0) \cdot\left[\operatorname{Im}_{\mathbb{E}_{1}}(Q)\right]\right) \\
& \quad \leq d_{\mathrm{H}}\left(\operatorname{Im}_{\mathbb{E}_{2}}\left(\varphi^{\mathbb{E}_{1}, \mathbb{E}_{2}}(Q)\right), \bar{\varphi}^{\mathbb{E}_{1}}(Q)\right)+d_{\mathrm{H}}\left(\bar{\varphi}^{\mathbb{E}_{1}}(Q), \varphi(0)+\frac{\partial \varphi}{\partial x}(0) \cdot\left[\operatorname{Im}_{\mathbb{E}_{1}}(Q)\right]\right) .
\end{aligned}
$$

By assumption, we have

$$
d_{\mathrm{H}}\left(\operatorname{Im}_{\mathbb{E}_{2}}\left(\varphi^{\mathbb{E}_{1}, \mathbb{E}_{2}}(Q)\right), \bar{\varphi}^{\mathbb{E}_{1}}(Q)\right)=\mathbf{O}\left(\left\|\operatorname{Im}_{\mathbb{E}_{1}}(Q)\right\|_{\mathrm{H}}^{2}\right),
$$

and, by Taylor's theorem, we also have

$$
d_{\mathrm{H}}\left(\bar{\varphi}^{\mathbb{E}_{1}}(Q), \varphi(0)+\frac{\partial \varphi}{\partial x}(0) \cdot\left[\operatorname{Im}_{\mathbb{E}_{1}}(Q)\right]\right)=\mathbf{O}\left(\left\|\operatorname{Im}_{\mathbb{E}_{1}}(Q)\right\|_{\mathrm{H}}^{2}\right) .
$$

2.3. Construction of set extensions of factorable functions. This subsection presents a systematic approach for constructing quadratically Hausdorff convergent extensions of twice continuously differentiable factorable functions when the underlying affine set-parameterization is invariant under affine transformation-see Definition 2.2. For simplicity of presentation, we only discuss the construction of an extension $\varphi^{\mathbb{E}_{\ell}}: \mathbb{D}_{n, \ell} \rightarrow \mathbb{D}_{m, \ell}$ of a given vector function $\varphi: \mathbb{R}^{n} \rightarrow \mathbb{R}^{m}$ subsequently, the construction of more general extensions acting on different basis sets being analogous.

The construction starts with a first-order Taylor expansion of the function $\varphi$ at a point $x^{*} \in \mathbb{R}^{n}$,

$$
\varphi(x)=\varphi\left(x^{*}\right)+A\left(x-x^{*}\right)+R\left(\xi_{1}, \ldots, \xi_{m}, x-x^{*}\right),
$$

where $R\left(\xi_{1}, \ldots, \xi_{m}, x-x^{*}\right):=\left(r_{1}\left(\xi_{1}, x-x^{*}\right), \ldots, r_{m}\left(\xi_{m}, x-x^{*}\right)\right)^{\mathrm{T}}$ and with the shorthand notation

$$
A:=\frac{\partial \varphi}{\partial x}\left(x^{*}\right) \quad \text { and } \quad \forall a, b \in \mathbb{R}^{n}, i \in\{1, \ldots, m\}, \quad r_{i}(a, b):=\left[\frac{\partial \varphi_{i}}{\partial x}(a)-A_{i}\right] b .
$$

Points $\xi_{i} \in \operatorname{conv}\left(\left\{x, x^{*}\right\}\right), i \in\{1, \ldots, m\}$, such that (2.4) holds, where $\operatorname{conv}\left(\left\{x, x^{*}\right\}\right)$ denotes the convex hull of the points $x$ and $x^{*}$ in $\mathbb{R}^{n}$, are guaranteed to exist by the mean-value theorem. Moreover, $\varphi$ being a factorable and continuously differentiable function, the forward mode of automatic differentiation can be applied and the residual function $R$ is itself a factorable function. At this point, it is worth noting that there is no unique way of choosing a suitable expansion point $x^{*}$. In the analysis that follows, we shall assume that $x^{*}$ is contained in the original set $\operatorname{Im}_{\mathbb{E}_{\ell}}(Q)$; for instance, the center of that set if $\operatorname{Im}_{\mathbb{E}_{\ell}}(Q)$ is an ellipsoid. Likewise, there are multiple ways of choosing $A$ besides the Jacobian of $\varphi$ at $x^{*}$, including the Jacobian of $\varphi$ at other points or the midpoint of an interval enclosure of the Jacobian of $\varphi$ on $\operatorname{Im}_{\mathbb{E}_{\ell}}(Q)$.

Provided that an extension $R^{\mathbb{E}_{\ell}}: \mathbb{D}_{n, \ell} \rightarrow \mathbb{D}_{m, \ell}$ of the remainder function $R$ is available such that

$$
\left\{R\left(\xi_{1}, \ldots, \xi_{m}, x-x^{*}\right) \mid \xi_{1}, \ldots, \xi_{m}, x \in \operatorname{Im}_{\mathbb{E}_{\ell}}(Q)\right\} \subseteq \operatorname{Im}_{\mathbb{E}_{\ell}}\left(R^{\mathbb{E}_{\ell}}(Q)\right),
$$

an extension of the function $\varphi$ can be obtained in the form

$$
\varphi^{\mathbb{E}_{\ell}}(Q):=\left(A Q+\left(0_{m \times \ell}, \varphi\left(x^{*}\right)\right)\right) \uplus R^{\mathbb{E}_{\ell}}(Q) .
$$


The following theorem provides conditions under which such an extension has quadratic Hausdorff convergence.

THEOREM 2.7. Let the affine set-parameterization $\left(\mathbb{E}_{\ell}, \mathbb{D}_{n, \ell}\right)$ be invariant under affine transformation and such that the addition extension $\uplus$ is regular. Assume that the extension $R^{\mathbb{E}_{\ell}}$ of the residual function $R$ in (2.4) is locally Lipschitz continuous, so that for all $Q \in \mathbb{D}_{n, \ell}$ with sufficiently small $\operatorname{diam}\left(\operatorname{Im}_{\mathbb{E}_{\ell}}(Q)\right)$, there exists a constant $L<\infty$ such that

$$
\left\|R^{\mathbb{E}_{\ell}}(Q)\right\|_{H} \leq L\left\|\left\{R\left(\xi_{1}, \ldots, \xi_{m}, x-x^{*}\right) \mid x, \xi_{1}, \ldots, \xi_{m} \in \operatorname{Im}_{\mathbb{E}_{\ell}}(Q)\right\}\right\|_{H}
$$

with $x^{*} \in \operatorname{Im}_{\mathbb{E}_{\ell}}(Q)$. Then, the extension $\varphi^{\mathbb{E}_{\ell}}$ in (2.5) has Hausdorff convergence order 2 .

Proof. Let $x^{*} \in \operatorname{Im}_{\mathbb{E}_{\ell}}(Q)$. By invariance of $\left(\mathbb{E}_{\ell}, \mathbb{D}_{n, \ell}\right)$ under affine transformation, the set $A Q+\left(0_{m \times \ell}, \varphi\left(x^{*}\right)\right)$ corresponds to the exact image of the affine approximation $\varphi\left(x^{*}\right)+A\left(x-x^{*}\right)$ of $\varphi$ on $\operatorname{Im}_{\mathbb{E}_{\ell}}(Q)$. Moreover, $\varphi$ being twice continuously differentiable, we have

$$
\max _{x, \xi_{1}, \ldots, \xi_{m} \in \operatorname{Im}_{\mathbb{E}_{\ell}}(Q)}\left\|R\left(\xi_{1}, \ldots, \xi_{m}, x-x^{*}\right)\right\|=\mathbf{O}\left(\left\|\operatorname{Im}_{\mathbb{E}_{\ell}}(Q)-x^{*}\right\|_{H}^{2}\right),
$$

and it follows from local Lipschitz-continuity of $R^{\mathbb{E}_{\ell}}$ that

$$
\left\|R^{\mathbb{E}_{\ell}}(Q)\right\|_{H}=\mathbf{O}\left(\left\|\operatorname{Im}_{\mathbb{E}_{\ell}}(Q)-x^{*}\right\|_{H}^{2}\right)
$$

for any $Q \in \mathbb{D}_{n, \ell}$ with sufficiently small $\operatorname{diam}\left(\operatorname{Im}_{\mathbb{E}_{\ell}}(Q)\right)$. The result follows by noting that the addition extension $\uplus$ is regular.

Although the proof of Theorem 2.7 is quite straightforward from a mathematical standpoint, the construct (2.5) proves especially useful to compute practical extensions of factorable functions with quadratic Hausdorff convergence. The key step involves constructing a Lipschitz-continuous range bounder of the residual function $R$, yet this poses no particular problem as simple interval arithmetics can be used for this purpose [23].

An arithmetic that meets all the requirements of Theorem 2.7, and therefore allows constructing quadratically Hausdorff convergent extensions, is based on Taylor models with ellipsoidal remainders as introduced in [38]. In particular, this arithmetic is used in the numerical case study of section 5. Further implementation details are given in Appendix B and [16].

3. Discretized set-valued integration algorithm. The focus in this section is on parametric nonlinear ODEs of the form (1.1), in connection to which we make the following blanket assumptions:

(A1) The right-hand side function $f: \mathbb{R} \times \mathbb{R}^{n_{x}} \times \mathbb{R}^{n_{p}} \rightarrow \mathbb{R}^{n_{x}}$ is jointly smooth in $(t, x, p)$ and factorable.

(A2) The initial-value function $x_{0}: \mathbb{R}^{n_{p}} \rightarrow \mathbb{R}^{n_{x}}$ is smooth in $p$ and factorable.

(A3) The parameter set $P$ is compact and a parameterization $Q_{p} \in \mathbb{D}_{n_{p}, \ell}$ is given such that $\operatorname{Im}_{\mathbb{E}_{\ell}}\left(Q_{p}\right) \supseteq P$, with $\ell \geq 1$ the actual number of degrees of freedom in the parameterization.

We note that Assumption A1 is mainly introduced to keep notation in the paper as simple as possible, and the algorithm presented later can be readily applied to more general classes of parametric ODEs, whose right-hand side functions are sufficiently often continuously differentiable. Likewise, Assumption A2 makes it possible to compute an enclosure of a (possibly nonconvex) initial value set of the form 
$X_{0}:=\left\{x_{0}(p) \mid p \in P\right\}$. In presenting the set-propagation algorithm, we keep our considerations general and do not specialize to a particular affine set-parameterization. Regarding existence and uniqueness of the ODE solutions, we note that a solution $x(\cdot, p):[0, T] \rightarrow \mathbb{R}^{n_{x}}$ of the initial-value problem (1.1) may not exist for all $p \in P$, but it is guaranteed to be unique on its maximum interval of existence by Assumption A1.

The objective of the following considerations is to develop a numerical algorithm that constructs a matrix valued function $Q_{x}:[0, T] \rightarrow \mathbb{D}_{n_{x}, \ell}$ such that

$$
\forall t \in[0, T], \quad \operatorname{Im}_{\mathbb{E}_{\ell}}\left(Q_{x}(t)\right) \supseteq X(t) .
$$

Similar to existing validated integrators for nonlinear ODEs [26, 27, 32], the proposed algorithm considers a Taylor series expansion in time of the ODE solutions. Assuming that $x(\cdot, p)$ is the solution of (1.1) up to time $t \in[0, T)$ for a given parameter $p$, and provided that this solution can be extended until $t+h$ with $h \in(0, T-t]$, the application of Taylor's theorem for an sth-order expansion gives

$$
x(t+h, p)=\sum_{i=0}^{s} h^{i} \phi_{i}(t, x(t, p), p)+h^{s+1} \phi_{s+1}(\tau, x(\tau, p), p)
$$

for some $\tau \in[t, t+h]$. Here, $\phi_{0}, \phi_{1}, \ldots, \phi_{s+1}:[0, T] \times \mathbb{R}^{n_{x}} \times \mathbb{R}^{n_{p}} \rightarrow \mathbb{R}^{n_{x}}$ denote the Taylor coefficient functions of the solution, defined recursively as

$$
\phi_{0}(t, x, p):=x \quad \text { and } \quad \phi_{i}(t, x, p):=\frac{1}{i}\left(\frac{\partial \phi_{i-1}}{\partial x}(t, x, p) f(t, x, p)+\frac{\partial \phi_{i-1}}{\partial t}(t, x, p)\right)
$$

for $i=1, \ldots, s+1$ and for all $(t, x, p) \in[0, T] \times \mathbb{R}^{n_{x}} \times \mathbb{R}^{n_{p}}$.

State-of-the-art validated integrators $[26,27,32]$ proceed in two phases, a first phase that determines a step-size and an a priori enclosure of the solution trajectory, followed by a tightening of this a priori enclosure in the second phase. In contrast, the algorithm presented below reverses the order of these two phases, thereby removing the need for an a priori enclosure of the solution and providing a natural mechanism for step-size selection. This procedure is described next for the propagation of a generic affine set-parameterization, as introduced in section 2. It starts with a parameterization $Q_{x}(0):=x_{0}^{\mathbb{E}_{\ell}}\left(Q_{p}\right)$, with $x_{0}^{\mathbb{E}_{\ell}}: \mathbb{D}_{n_{p}, \ell} \rightarrow \mathbb{D}_{n_{x}, \ell}$ an extension of the initial-value function $x_{0}$, so that $\operatorname{Im}_{\mathbb{E}_{\ell}}\left(Q_{x}(0)\right) \supseteq X(0)$. Then, the following two steps are applied repeatedly:

Step 1. Given a parameterization $Q_{x}(t)$ at some $t \in[0, T)$ such that $\operatorname{Im}_{\mathbb{E}_{\ell}}\left(Q_{x}(t)\right) \supseteq$ $X(t)$, a predictor $Q_{x}(t+h)$ of the solution for all $h \in(0, T-t]$ is given by

$$
Q_{x}(t+h):=\biguplus_{i=0}^{s} h^{i} \phi_{i}^{\mathbb{E}_{\ell}}\left(t, Q_{x}(t), Q_{p}\right) \uplus h \mathrm{TOL} Q_{\text {unit }}
$$

for a prespecified tolerance TOL $>0$ and a given $Q_{\text {unit }} \in \mathbb{D}_{n_{x}, \ell}$ to be defined below, and where $\phi_{i}^{\mathbb{E}_{\ell}}:[0, T] \times \mathbb{D}_{n_{x}, \ell} \times \mathbb{D}_{n_{p}, \ell} \rightarrow \mathbb{D}_{n_{x}, \ell}$ are extensions of the Taylor coefficient functions $\phi_{i}$ for each $i=0, \ldots, s$ and $\uplus$ stands for the addition extension according to Definition 2.4. In order to avoid confusion at this point, we note that the predictor $Q_{x}(t+h)$ does not need to be evaluated for a particular step-size during the propagation. In a practical implementation, $Q_{x}$ can be stored in the form a computational graph, with one of the graph leaves corresponding to the (symbolic) variable $h$. This way, an enclosure $\operatorname{Im}_{\mathbb{E}_{\ell}}\left(Q_{x}(t+h)\right) \supseteq X(t+h)$ can always be obtained after the propagation, by evaluating (3.2) for any step-size $h$ satisfying the validation condition given next. 
Step 2. A step-size $\bar{h}$ is determined such that the predictor $Q_{x}(t+h)$ in $(3.2)$ is guaranteed to yield a valid enclosure of the reachable set, $\operatorname{Im}_{\mathbb{E}_{\ell}}\left(Q_{x}(t+h)\right) \supseteq$ $X(t+h)$, for all $h \in[0, \bar{h}]$. In particular, any feasible point $\bar{h}>0$ of the following optimization problem is suitable:

$$
\sup _{h>0} h \text { s.t. }\left\{\begin{array}{l}
\forall \tau \in[t, t+h], \\
\operatorname{Im}_{\mathbb{E}_{\ell}}\left((\tau-t)^{s} \phi_{s+1}^{\mathbb{E}_{\ell}}\left(t, Q_{x}(\tau), Q_{p}\right)\right) \subseteq \operatorname{TOL} \operatorname{Im}_{\mathbb{E}_{\ell}}\left(Q_{\text {unit }}\right)
\end{array}\right.
$$

with $\phi_{s+1}^{\mathbb{E}_{\ell}}:[0, T] \times \mathbb{D}_{n_{x}, \ell} \times \mathbb{D}_{n_{p}, \ell} \rightarrow \mathbb{D}_{n_{x}, \ell}$ an extension of the Taylor coefficient function $\phi_{s+1}$.

The validity of this bounding procedure is established in the following theorem.

THEOREM 3.1. Let the function $f$ satisfy the blanket assumption $\mathrm{A} 1$, and let $Q_{x}(t) \in \mathbb{D}_{n_{x}, \ell}$ be such that $\operatorname{Im}_{\mathbb{E}_{\ell}}\left(Q_{x}(t)\right) \supseteq X(t)$. If $\bar{h}$ is a feasible point of the stepsize selection problem (3.3), then $\operatorname{Im}_{\mathbb{E}_{\ell}}\left(Q_{x}(t+h)\right) \supseteq X(t+h)$ for all $h \in[0, \bar{h}]$, with $Q_{x}(t+h)$ given by $(3.2)$.

Proof. It follows from the application of affine set arithmetic to the Taylor series expansion (3.1) that $\operatorname{Im}_{\mathbb{E}_{\ell}}\left(Q_{x}(t+h)\right) \supseteq X(t+h)$ whenever the remainder term $(\tau-$ $t)^{s+1} \phi_{s+1}(x(\tau, p))$ is contained in the set $\operatorname{Im}_{\mathbb{E}_{\ell}}\left(h \mathrm{TOL} Q_{\text {unit }}\right)$ for all $\tau \in[t, t+h]$. The semi-infinite constraint in (3.3) ensures that the remainder term satisfies this condition on the interval $[0, \bar{h}]$ by construction.

For any practical purposes, it is convenient (and sufficient) to solve the stepsize selection problem (3.3) approximately. Consider the remainder function $r_{t}$ : $[0, T-t] \rightarrow \mathbb{D}_{n_{x}}^{\text {interval }}$ given by

$$
r_{t}(h):=B_{n_{x}}\left(\phi_{s+1}^{\mathbb{E}_{\ell}}\left(t, Q_{x}(t+h), Q_{p}\right)\right),
$$

where $B_{n_{x}}: \mathbb{D}_{n_{x}, \ell} \rightarrow \mathbb{D}_{n_{x}}^{\text {interval }}$ is a range bounder for the chosen affine set-parameterization,

$$
\forall Q \in \mathbb{D}_{n_{x}, \ell}, \quad \operatorname{Im}_{\mathbb{E}^{\ell}}(Q) \subseteq \operatorname{Im}_{\mathbb{E}_{n_{x}}^{\text {box }}}\left(B_{n_{x}}(Q)\right)
$$

and let $r_{t}^{\mathbb{E}_{1}^{\text {box }}, \mathbb{E}_{n_{x}}^{\text {box }}}: \mathbb{D}_{1}^{\text {interval }} \rightarrow \mathbb{D}_{n_{x}}^{\text {interval }}$ be an interval extension of $r_{t}$. The following corollary of Theorem 3.1 is immediate upon noting that

$$
\operatorname{Im}_{\mathbb{E}_{\ell}}\left(h^{s} \phi_{s+1}^{\mathbb{E}_{\ell}}\left(t, Q_{x}(t+h), Q_{p}\right)\right) \subseteq \operatorname{Im}_{\mathbb{E}_{n_{x}}^{\text {box }}}\left(r_{t}^{\mathbb{E}_{1}^{\text {box }}, \mathbb{E}_{n_{x}}^{\text {box }}}\left(\frac{1}{2} h, \frac{1}{2} h\right)\right)
$$

for all $h \in[0, T-t]$.

COROLlary 3.2. Let the function $f$ satisfy the blanket assumption A1, let $Q_{x}(t) \in \mathbb{D}_{n_{x}, \ell}$ be such that $\operatorname{Im}_{\mathbb{E}_{\ell}}\left(Q_{x}(t)\right) \supseteq X(t)$, and let $\sigma \in \mathbb{R}_{+}^{n_{x}}, \sigma>0$, be such that $[-\sigma, \sigma] \subseteq \operatorname{Im}_{\mathbb{E}_{\ell}}\left(Q_{\text {unit }}\right)$. Suppose that $\bar{h} \in(0, T-t]$ satisfies

$$
\bar{h}^{s}\left\|\operatorname{diag}(\sigma)^{-1} \operatorname{abs}\left(\operatorname{Im}_{\mathbb{E}_{n_{x}}^{\text {box }}}\left(r_{t}^{\mathbb{E}_{1}^{\text {box }}, \mathbb{E}_{n_{x}}^{\text {box }}}\left(\frac{1}{2} h, \frac{1}{2} h\right)\right)\right)\right\|_{\infty} \leq \text { TOL } .
$$

Then, $\operatorname{Im}_{\mathbb{E}_{\ell}}\left(Q_{x}(t+h)\right) \supseteq X(t+h)$ for all $h \in[0, \bar{h}]$, with $Q_{x}(t+h)$ given by (3.2).

In particular, (3.4) provides a practical condition for step-size validation. The following simple iterative procedure determines a feasible step-size: 
1. Consider the following initial guess for the step-size:

$$
\bar{h}=\rho\left(\frac{\mathrm{TOL}}{\left\|\operatorname{diag}(\sigma)^{-1} \operatorname{abs}\left(\operatorname{Im}_{\mathbb{E}_{n_{x}}^{\text {box }}}\left(r_{t}^{\mathbb{E}_{1}^{\text {box }}, \mathbb{E}_{n_{x}}^{\text {box }}}(0)\right)\right)\right\|_{\infty}}\right)^{\frac{1}{s}}
$$

with $0<\rho<1$ a tuning parameter, e.g., $\rho=0.8$.

2. While condition (3.4) is not satisfied with $\bar{h}$, reduce the step-size as $\bar{h} \leftarrow \rho \bar{h}$. Note that this procedure is guaranteed to identify a feasible step-size $\bar{h}>0$ after finitely many iterations, since the left-hand side expression in (3.4) shrinks with order $O\left(h^{s}\right)$, whereas the right-hand term is constant and has a nonempty interior.

As far as the selection of $Q_{\text {unit }}$ and $\sigma$ is concerned, a practical procedure involves setting $\sigma$ first - e.g., by accounting for the relative magnitude of the state variablesand determining $Q_{\text {unit }}$ accordingly, in such a way that $\operatorname{Im}_{\mathbb{E}_{\ell}}\left(Q_{\text {unit }}\right) \supseteq[-\sigma, \sigma]$. In analogy to standard scaling heuristics used in state-of-the-art (nonvalidated) ODE solvers [11], $\sigma$ can be seen as a scaling vector and adjusted dynamically during the integration as follows:

$$
\sigma_{i}:=\frac{1}{2} B_{n_{x}}\left(Q_{x}(t)\right)_{i, i}+\frac{\mathrm{ATOL}}{\mathrm{TOL}}, \quad i=1, \ldots, n_{x},
$$

where ATOL $>0$ is an additional tuning parameter, named absolute tolerance.

The full discretized set-valued integration procedure is summarized in Algorithm 1. Note that this algorithm terminates with an error message as soon as the existence of the reachable set $X(t)$ can no longer be established. Such scenarios cannot be avoided, for instance, if a solution trajectory $x(\cdot, p)$ fails to exist over the entire horizon $[0, T]$ for certain parameters $p \in P$ or if the enclosure size blows up due to wrapping effects.

Algorithm 1. Set-VAlued integration of a PARAmetric initial VAlue ProbLEM IN ODES FOR A GENERIC AFFINE SET-PARAMETERIZATION.

Input: ODE (1.1) with factorable right-hand side $f$ and initial value function $x_{0}$; affine parameterization $Q_{p} \in \mathbb{D}_{n_{p}, \ell}$ such that $\operatorname{Im}_{\mathbb{E}_{\ell}}\left(Q_{p}\right) \supseteq P$; consistency order $s \geq 1$; tolerances TOL $\geq$ ATOL $>0$; maximum and minimum step-sizes $h_{\max } \geq h_{\min }>0$; step-size reduction parameter $0<\varrho<1$.

Initialization:

1. Set $t=0$, and $Q_{x}(t)=x_{0}^{\mathbb{E}_{\ell}}\left(Q_{p}\right)$.

\section{Loop:}

2. Adjust the scaling vector $\sigma$ using (3.6).

3. Set the predictor $Q_{x}(t+h)$ for all $h \in[0, T-t]$ as in (3.2).

4. Set the step-size guess $h:=\min \left\{\bar{h}, T-t, h_{\max }\right\}$, with $\bar{h}$ given in (3.5). While condition (3.4) is violated, repeat $h \leftarrow \varrho h$.

5. If $h<h_{\min }$, return with an error message.

6. Update $t \leftarrow t+h$

7. If $t+h=T$, return with an indication of completion; else return to step 2

Output: Enclosure function $Q_{x}:[0, t] \rightarrow \mathbb{D}_{n_{x}, \ell}$ such that $\operatorname{Im}_{\mathbb{E}_{\ell}}\left(Q_{x}(\tau)\right) \supseteq X(\tau)$ for all $\tau \in[0, t]$, with $t \leq T$. 
On the other hand, not only does Algorithm 1 yield a valid enclosure of the ODE solution upon successful termination, but it also provides a guarantee that the solution trajectories $x(\cdot, p)$ exist for all $p \in P \subseteq \operatorname{Im}_{\mathbb{E}_{\ell}}\left(Q_{p}\right)$.

4. Stability analysis. Conditions under which the discretized set-valued integration algorithm presented above inherits the stability properties of the underlying dynamic system are investigated in this section. Of particular interest are those parametric ODEs having a unique equilibrium point $\bar{x}(p)$ for every $p \in P$. More generally, we shall consider the case that the function $f(\cdot, x, p)$ is periodic and satisfies the following assumption.

Assumption 1. The parametric ODEs (1.1) have a unique asymptotically stable limit cycle $\bar{x}(\cdot, p)$ for every $p \in P$ such that

$$
\forall t \in[0, T], \quad \dot{\bar{x}}(t, p)=f(t, \bar{x}(t, p), p), \quad \text { and } \quad \bar{x}(0, p)=\bar{x}(T, p)
$$

for some $T>0$.

Implicit to condition (4.1) is the requirement that the cycle time $T$ should be identical for all $p \in P$, thereby restricting the class of periodic dynamic systems that are considered here. Conditions under which $T$ is independent of the time $t$, at least locally, are further discussed in Appendix A.

Example 6. Consider the scalar differential equation

$$
\dot{x}(t)=-p_{1} x(t)+\sin \left(p_{2} t\right)+p_{3}
$$

with parameters $p_{1}>0, p_{2} \neq 0$, and $p_{3} \in \mathbb{R}$. The following function

$$
\bar{x}(t, p):=\frac{1}{p_{1}}\left[\left(1-\frac{p_{2}^{2}}{p_{1}}\right)\left(\sin \left(p_{2} t\right)-\frac{p_{2}}{p_{1}} \cos \left(p_{2} t\right)\right)+p_{3}\right]
$$

satisfies condition (4.1) with the cycle time $T=\frac{2 \pi}{p_{2}}$. In particular, the cycle time $T$ is constant if parameter $p_{2}$ is fixed. On the other hand, Assumption 1 fails to hold when $p_{2}$ is allowed to vary.

Given a compact parameter host set $P \subset \mathbb{R}^{n_{p}}$, Algorithm 1 propagates a parameterization $Q_{x}(t, P) \in \mathbb{D}_{n_{x}, \ell}$, whose image describes a valid enclosure of the reachable parametric initial value problem (1.1), $\operatorname{Im}_{\mathbb{E}_{\ell}}\left(Q_{x}(t, P)\right) \supseteq X(t, P)$, for $t \geq 0$. In analogy with the definition of local asymptotic stability in dynamic systems, we formalize the concept of local asymptotic stability for a set-valued integration algorithm next.

Definition 4.1. Let Assumption 1 be satisfied, and denote $\bar{X}(t, P):=\{\bar{x}(t, p) \mid$ $p \in P\}$ for $t \geq 0$. Algorithm 1 for set-valued integration with consistency order $s \geq 1$ is said to be locally asymptotically stable for the parametric ODE (1.1) if the following conditions are satisfied for sufficiently small local tolerance TOL $>0$ and maximum step-size $h_{\max }>0$ :

(i) For every $\epsilon>0$ and all $t \geq 0$, there exists $\delta>0$ such that

$$
d_{\mathrm{H}}\left(\operatorname{Im}_{\mathbb{E}_{\ell}}\left(Q_{x}(t, P)\right), \bar{X}(t, P)\right)=\epsilon+\mathbf{O}(\mathrm{TOL})+\mathbf{O}\left(h_{\text {max }}^{s}\right)
$$

for all $P \subseteq \mathbb{R}^{n_{p}}$ with $\operatorname{diam}(P)<\delta$ and all $Q_{x}(0, P)$ with $d_{\mathrm{H}}\left(\operatorname{Im}_{\mathbb{E}_{\ell}}\left(Q_{x}(0, P)\right), \bar{X}(0, P)\right)$ $<\delta$.

(ii) There exists $\delta>0$ such that

$$
\limsup _{t \rightarrow \infty}\left\|\operatorname{Im}_{\mathbb{E}_{\ell}}\left(Q_{x}\left(t,\left\{p^{*}\right\}\right)\right)-\bar{x}\left(t, p^{*}\right)\right\|_{\mathrm{H}}=\mathbf{O}(\mathrm{TOL})+\mathbf{O}\left(h_{\max }^{s}\right)
$$

for all $p^{*} \in \mathbb{R}^{n_{p}}$ and all $Q_{x}\left(0,\left\{p^{*}\right\}\right)$ with $\left\|\operatorname{Im}_{\mathbb{E}_{\ell}}\left(Q_{x}\left(0,\left\{p^{*}\right\}\right)\right)-\bar{x}\left(0, p^{*}\right)\right\|_{\mathrm{H}}<\delta$. 
A key advantage of a locally asymptotically stable set-valued integrator (in the sense of Definition 4.1) is that the computed reachable set enclosures are guaranteed to remain stable on infinite time horizons when applied to a dynamic system in the neighborhood of a locally asymptotically stable periodic orbit (or locally asymptotically stable equilibrium point). Moreover, in the case that the only parametric uncertainty is via the initial condition, the enclosure $\operatorname{Im}_{\mathbb{E}_{\ell}}\left(Q_{x}\left(t,\left\{p^{*}\right\}\right)\right)$ converges to the periodic orbit $\bar{X}\left(t,\left\{p^{*}\right\}\right)$ for any given parameter $p^{*}$, up to a small numerical "noise" of order $\mathbf{O}(\mathrm{TOL})+\mathbf{O}\left(h_{\max }^{s}\right)$ that can be made arbitrarily small by adjusting the tuning parameters TOL and $h_{\max }$ in Algorithm 1.

Remark 3. In an alternative definition of local asymptotic stability of a set-valued integrator, the term $\mathbf{O}\left(h_{\max }^{s}\right)$ could be dropped in (4.2) and (4.3), thereby accounting for terms of order $\mathbf{O}$ (TOL) only. From a practical viewpoint, this refinement would be more appropriate for stiff dynamic systems, where a larger $h_{\max }$ is desirable in order to not slow down the integration process unnecessarily. The emphasis in this paper being on explicit integration schemes, the assumption is made that a small $h_{\max }$ value compared to the characteristic timescales of the dynamics should not have a major impact on the performance of the integration algorithm, at least for small TOL values. We shall revisit this important aspect later on in Remark 4, once we have developed a better understanding of the mechanisms that may lead to instability in Algorithm 1.

Although asymptotic stability of the computed reachable set enclosures would appear to be a natural property for any set-valued integration algorithm to have, all currently available set-valued integrators lack it to the best of our knowledge. This could be attributed to the fact that these integrators rely on interval arithmetics in one way or another and are thus subject to bound explosion in finite time due to wrapping effects, regardless of the size of the uncertainty set. Even state-of-the-art integrators based on Taylor models with interval remainders, such as VSPODE [18], cannot prevent bound explosion in enclosing the reachable set of asymptotically stable dynamic systems, despite the fact that they implement advanced heuristics for rotating the basis of the interval remainder.

In order to determine conditions under which a set-valued integration algorithm is asymptotically stable, we first recall basic stability results for periodic dynamic systems; see, e.g., [37] for more details. Given a parametric ODE of the form (1.1) and a limit cycle $\bar{x}(\cdot, p)$ satisfying (4.1) for $p \in P$, the so-called monodromy matrix $\Phi(T, 0, p)$ can be obtained as the solution of the variational differential equation for $\tau, t \in[0, T]:$

$$
\frac{\partial \Phi(t, \tau, p)}{\partial t}=\frac{\partial f(t, \bar{x}(t, p), p)}{\partial x} \Phi(t, \tau, p) \quad \text { with } \quad \Phi(\tau, \tau, p)=I .
$$

It can be shown that the periodic orbit $\bar{x}(\cdot, p)$ is locally asymptotically stable if all the eigenvalues $\lambda_{i}(G(T, 0, p))$ of the monodromy matrix are in the open unit disk,

$$
\max _{i \in\left\{1, \ldots, n_{x}\right\}}\left|\lambda_{i}(\Phi(T, 0, p))\right|<1 .
$$

The following theorem establishes quadratic Hausdorff convergence of the underlying affine set-parameterization to be the critical requirement for local asymptotic stability of Algorithm 1.

THEOREM 4.2. Let Assumption 1 hold, and consider an affine set-parameterization for which (i) function extensions have Hausdorff convergence order 2 (or higher), and 
(ii) the addition extension is consistent in the sense of Definition 2.4. Then, Algorithm 1 with consistency order $s \geq 1$ is locally asymptotically stable for the parametric ODE (1.1).

Proof. Let $\bar{x}(\cdot, p)$ satisfy condition (4.1) in Assumption 1 for a given $p \in P$ and be a locally asymptotically stable orbit. For any $t \in[0, T)$, any $Q_{p}(P) \in \mathbb{D}_{n_{p}, \ell}$ with sufficiently small $\left\|\operatorname{Im}_{\mathbb{E}_{\ell}}\left(Q_{p}(P)\right)-p\right\|_{\mathrm{H}}$, any $Q_{x}(t, P) \in \mathbb{D}_{n_{x}, \ell}$ with sufficiently small $\left\|\operatorname{Im}_{\mathbb{E}_{\ell}}\left(Q_{x}(t, P)\right)-\bar{x}(t, p)\right\|_{\mathrm{H}}$, and any step-size $h$ satisfying condition (3.4), we have

$$
\begin{aligned}
& \left\|\operatorname{Im}_{\mathbb{E}_{\ell}}\left(Q_{x}(t+h, P)\right)-\bar{x}(t+h, p)\right\|_{\mathrm{H}} \\
& \stackrel{(3.2)}{=}\left\|\operatorname{Im}_{\mathbb{E}_{\ell}}\left(\biguplus_{i=0}^{s} h^{i} \phi_{i}^{\mathbb{E}_{\ell}}\left(t, Q_{x}(t, P), Q_{p}(P)\right) \oplus h \mathrm{TOL} Q_{\mathrm{unit}}\right)-\bar{x}(t+h, p)\right\|_{\mathrm{H}} \\
& \stackrel{(2.2)}{=}\left\|\operatorname{Im}_{\mathbb{E}_{\ell}}\left(\biguplus_{i=0}^{s} h^{i} \phi_{i}^{\mathbb{E} \ell}\left(t, Q_{x}(t, P), Q_{p}(P)\right)\right)-\sum_{i=0}^{s} h^{i} \phi_{i}(\bar{x}(t, p))\right\|_{\mathrm{H}}+h \mathbf{O}(\mathrm{TOL}) .
\end{aligned}
$$

Since the Taylor coefficient functions are continuously-differentiable and their extension have quadratic Hausdorff convergence, Proposition 2.6 gives

$$
\begin{aligned}
\| \operatorname{Im}_{\mathbb{E}_{\ell}} & \left(Q_{x}(t+h, P)\right)-\bar{x}(t+h, p) \|_{\mathrm{H}} \\
\stackrel{(2.3)}{=} & \left\|\sum_{i=0}^{s} h^{i} \frac{\partial \phi_{i}}{\partial x}(t, \bar{x}(t, p), p) \cdot\left[\operatorname{Im}_{\mathbb{E}_{\ell}}\left(Q_{x}(t, P)\right)-\bar{x}(t, p)\right]\right\|_{\mathrm{H}} \\
& +\left\|\sum_{i=0}^{s} h^{i} \frac{\partial \phi_{i}}{\partial p}(t, \bar{x}(t, p), p) \cdot\left[\operatorname{Im}_{\mathbb{E}_{\ell}}\left(Q_{p}(P)\right)-p\right]\right\|_{\mathrm{H}}+h \mathbf{O}(\mathrm{TOL}) \\
& +h \mathbf{O}\left(\left\|\operatorname{Im}_{\mathbb{E}_{\ell}}\left(Q_{x}(t, P)\right)-\bar{x}(t, p)\right\|_{\mathrm{H}}^{2}\right)+h \mathbf{O}\left(\left\|\operatorname{Im}_{\mathbb{E}_{\ell}}\left(Q_{p}(P)\right)-p\right\|_{\mathrm{H}}^{2}\right) .
\end{aligned}
$$

Notice the absence of terms of order $\mathbf{O}\left(\left\|\operatorname{Im}_{\mathbb{E}_{\ell}}\left(Q_{x}(t, P)\right)-\bar{x}(t, p)\right\|_{\mathrm{H}}^{2}\right)$ or $\mathbf{O}\left(\left\|\operatorname{Im}_{\mathbb{E}_{\ell}}\left(Q_{p}(P)\right)-p\right\|_{\mathrm{H}}^{2}\right)$ in the right-hand side of the previous inequality-because the Taylor coefficient function $\phi_{0}$ is affine, its extension $\phi_{0}^{\mathbb{E}_{\ell}}$ matches the actual image set of $\phi_{0}$ exactly, and therefore all nontrivial terms are of order $\mathbf{O}(h)$. Now, by using a Taylor expansion of the solution $\Phi$ of the variational differential equation (4.4), we have

$$
\begin{aligned}
\| & {\left[\sum_{i=0}^{s} h^{i} \frac{\partial \phi_{i}}{\partial x}(t, \bar{x}(t, p), p)-\Phi(t+h, t, \bar{p})\right] \cdot\left[\operatorname{Im}_{\mathbb{E}_{\ell}}\left(Q_{x}(t, P)\right)-\bar{x}(t, p)\right] \|_{\mathrm{H}} } \\
& =h \mathbf{O}\left(\left\|\operatorname{Im}_{\mathbb{E}_{\ell}}\left(Q_{x}(t, P)\right)-\bar{x}(t, p)\right\|_{\mathrm{H}}\right)+\mathbf{O}\left(h^{s+1}\right) \\
& =h \mathbf{O}(\operatorname{diam}(P))+h \mathbf{O}\left(h_{\max }^{s}\right) .
\end{aligned}
$$

In the last inequality, we have used that $\operatorname{Im}_{\mathbb{E}_{\ell}}\left(Q_{x}(t, P)\right)$ is locally Lipschitz continuous in $P$, which follows trivially from the fact that extensions have quadratic Hausdorff convergence in the chosen set arithmetic. Moreover, we also have

$$
\begin{aligned}
& \left\|\sum_{i=0}^{s} h^{i} \frac{\partial \phi_{i}}{\partial p}(t, \bar{x}(t, p), p) \cdot\left[\operatorname{Im}_{\mathbb{E}_{\ell}}\left(Q_{p}(P)\right)-p\right]\right\|_{\mathrm{H}} \\
& \quad=h \mathbf{O}\left(\left\|\operatorname{Im}_{\mathbb{E}_{\ell}}\left(Q_{p}(P)\right)-p\right\|_{\mathrm{H}}\right)=h \mathbf{O}(\operatorname{diam}(P)) .
\end{aligned}
$$


Thus, combining (4.6), (4.7), and (4.8) gives

$$
\begin{aligned}
\left\|\operatorname{Im}_{\mathbb{E}_{\ell}}\left(Q_{x}(t+h, P)\right)-\bar{x}(t+h, p)\right\|_{\mathrm{H}} \\
=\left\|\Phi(t+h, t, p) \cdot\left[\operatorname{Im}_{\mathbb{E}_{\ell}}\left(Q_{x}(t, P)\right)-\bar{x}(t, p)\right]\right\|_{\mathrm{H}} \\
\quad+h \mathbf{O}\left(\left\|\operatorname{Im}_{\mathbb{E}_{\ell}}\left(Q_{x}(t, P)\right)-\bar{x}(t, p)\right\|_{\mathrm{H}}^{2}\right)+h \mathbf{O}\left(\left\|\operatorname{Im}_{\mathbb{E}_{\ell}}\left(Q_{p}(P)\right)-p\right\|_{\mathrm{H}}^{2}\right) \\
\quad+h \mathbf{O}(\operatorname{diam}(P))+h \mathbf{O}(\mathrm{TOL})+h \mathbf{O}\left(h_{\max }^{s}\right)
\end{aligned}
$$

for all $t \in[0, T]$.

In a second step, we apply a discrete version of Gronwall's lemma to obtain

$$
\begin{aligned}
&\left\|\operatorname{Im}_{\mathbb{E}_{\ell}}\left(Q_{x}(t+T, P)\right)-\bar{x}(t, p)\right\|_{\mathrm{H}} \\
&=\left\|\Phi(t+T, t, p) \cdot\left[\operatorname{Im}_{\mathbb{E}_{\ell}}\left(Q_{x}(t, P)\right)-\bar{x}(t, p)\right]\right\|_{\mathrm{H}} \\
&+\mathbf{O}\left(\left\|\operatorname{Im}_{\mathbb{E}_{\ell}}\left(Q_{x}(t, P)\right)-\bar{x}(t, p)\right\|_{\mathrm{H}}^{2}\right)+\mathbf{O}\left(\left\|\operatorname{Im}_{\mathbb{E}_{\ell}}\left(Q_{p}(P)\right)-p\right\|_{\mathrm{H}}^{2}\right) \\
&+\mathbf{O}(\operatorname{diam}(P))+\mathbf{O}(\mathrm{TOL})+\mathbf{O}\left(h_{\max }^{s}\right),
\end{aligned}
$$

since we can substitute $\bar{x}(t+T, p)=\bar{x}(t, p)$.

In the last step, we use induction over the number $N$ of cycle times together with the fact that $\lim _{N \rightarrow \infty} \Phi(t+T, t, p)^{N}=0$ for an asymptotically stable orbit in order to show that the above inequality implies

$$
\begin{aligned}
& \forall t \in[0, T], \quad \lim _{N \rightarrow \infty} d_{\mathrm{H}}\left(\operatorname{Im}_{\mathbb{E}_{\ell}}\left(Q_{x}(t+N T, P)\right), \bar{X}(t+N T, P)\right) \\
&=\mathbf{O}(\operatorname{diam}(P))+\mathbf{O}(\mathrm{TOL})+\mathbf{O}\left(h_{\max }^{s+1}\right) .
\end{aligned}
$$

In particular, (4.9) implies both conditions (i) and (ii) in Definition 4.1.

Theorem 4.2 sheds light on the most fundamental reason why existing set-valued integrators based on Taylor models (with interval remainders) fail to stabilize the computed reachable set enclosures for small enough uncertainty set, as these affine set-parameterizations have Hausdorff convergence order 1 in general (see Remark 2).

We also note that a set-valued integration algorithm may not inherit the stability properties of those dynamic systems that are locally stable, but not locally asymptotically stable. When invariants are known explicitly for such systems though, e.g., based on the underlying conservation laws in the case of physical systems, these invariants can sometimes be used to formulate an equivalent, reduced dynamic system that is locally asymptotically stable.

Finally, it is worth noting that the foregoing stability analysis can be extended to the case that the initial set $X_{0}=\left\{x_{0}(p) \mid p \in P\right\}$ is not necessarily close to the periodic orbit. More specifically, if for each $p \in P$ the response trajectory $x(\cdot, p)$ reaches an attractive neighborhood of the periodic limit orbit $\bar{X}(t, P)$ after a finite transition time, then Algorithm 1 shall remain stable on infinite horizons as long as the diameter of the parameter host set $P$ is sufficiently small.

Remark 4. As a final note in connection to Remark 3, it is interesting to observe that the term $\mathbf{O}\left(h_{\max }^{s}\right)$ is introduced in (4.8). The reason that the discretization error of the variational equation cannot be bounded with a term of order $\mathbf{O}$ (TOL) is because the step-size control mechanism in Algorithm 1, although it accounts for discretization errors in the nominal state trajectories rigorously, does not account for discretization errors in the associated variational equation on the other hand. In particular, this problem could be resolved by appending the following semi-infinite 
constraint to the step-size optimization problem (3.3):

$$
\forall \tau \in[t, t+h], \quad \operatorname{Im}_{\mathbb{E}_{\ell}}\left((\tau-t)^{s}{\frac{\partial \phi_{s+1}}{\partial x}}^{\mathbb{E}_{\ell}}\left(Q_{x}(\tau)\right)\right) \subseteq \operatorname{TOL} \operatorname{Im}_{\mathbb{E}_{\ell}}\left(Q_{\text {unit }}^{\prime}\right)
$$

for a suitable matrix $Q_{\text {unit }}^{\prime} \in \mathbb{D}_{n_{x} \times n_{x}, \ell}$. However, drawbacks of this approach include the need to compute enclosures of the Jacobian matrix of $\phi_{s+1}$, which can be computationally demanding in practice, as well as the extra conservatism it would introduce on the selected step-size. A potentially more efficient way of enforcing stability irrespective of the step-size control mechanism would involve developing implicit schemes for set-valued numerical ODE integration, which will be the topic for further research.

5. Case study: Cubic oscillator. The main objective of the numerical case study in this final section is merely to illustrate the ability of the developed setvalued ODE integrator to stabilize the reachable set enclosures - not to provide a detailed computational study. Our implementation of Algorithm 1 comes in the form of a C++ class called ODEBND_VAL as part of the CRONOS library, which is made freely available at http://omega-icl.bitbucket.org/cronos/. It uses the affine set-parameterization $\left(\mathbb{E}_{\ell}^{\mathrm{pol}(q)} \times \mathbb{E}_{n_{x}}^{\mathrm{ball}}, \mathbb{R}^{n_{x} \times\left(\alpha_{\ell}^{(q)}+n_{x}+1\right)}\right)$ of polynomial models combined with ellipsoids and computes quadratically Hausdorff convergent extensions based on Theorem 2.7; see Appendix B and [16] for more details on the construction of Taylor models with ellipsoidal remainder. In particular, we use the verified library PROFIL (http://www.ti3.tu-harburg.de/) for interval analysis and the library MC++ (http://omega-icl.bitbucket.org/mcpp/) [22] for Taylor model arithmetic. The Taylor expansion in time of the ODE solutions are constructed using automatic differentiation in FADBAD++ (http://www.fadbad.com/fadbad.html). Unless otherwise noted, the consistency order is set to $s=5$, the tolerances to TOL $=10^{-7}$ and ATOL $=10^{-8}$, and the step-size control parameter to $\rho=0.8$.

We consider the following cubic oscillator system:

$$
\begin{array}{ll}
\dot{x}_{1}(t)=x_{2}(t)+\frac{1}{10}\left(1-x_{1}(t)^{2}-x_{2}(t)^{2}\right) x_{1}(t) & \text { with } x_{1}(0)=p_{1}, \\
\dot{x}_{2}(t)=-x_{1}(t)+\frac{1}{10}\left(1-x_{1}(t)^{2}-x_{2}(t)^{2}\right) x_{2}(t)-\frac{1}{5} x_{2}(t) & \text { with } \quad x_{2}(0)=p_{2},
\end{array}
$$

where both parameters $p_{1}$ and $p_{2}$ are uncertain, given by $p \in P:=[1.5,3] \times[-0.1,0.1] \subseteq$ $\mathbb{R}^{2}$.

The results obtained by running the set-valued integrator with fourth-order Taylor models are given in Figure 1, with projections on the state components $x_{1}(t)$ and $x_{2}(t)$ shown on the left plot and the right plot, respectively. Both plots illustrate that the computed set $\operatorname{Im}_{\mathbb{E}_{\ell}^{\text {pol }(4)} \times \mathbb{E}_{2}^{\text {ball }}}\left(Q_{x}(\cdot, P)\right)$ validly enclose the actual reachable set on the time horizon $[0,20]$, with very small overestimation in this case.

The behavior of the set-valued integrator on the extended time horizon $t \in[0,400]$ is shown in Figure 2. Also reported on this figure are the bounds computed using VSPODE (v1.4) [18], with the order of the Taylor expansion in time and of the Taylor model set to 5 and 4 , respectively, in order to enable direct comparison, and selecting QR-factorization with row permutation as the wrapping mitigation strategy. These results demonstrate the ability of Algorithm 1 to stabilize the reachable set enclosures 

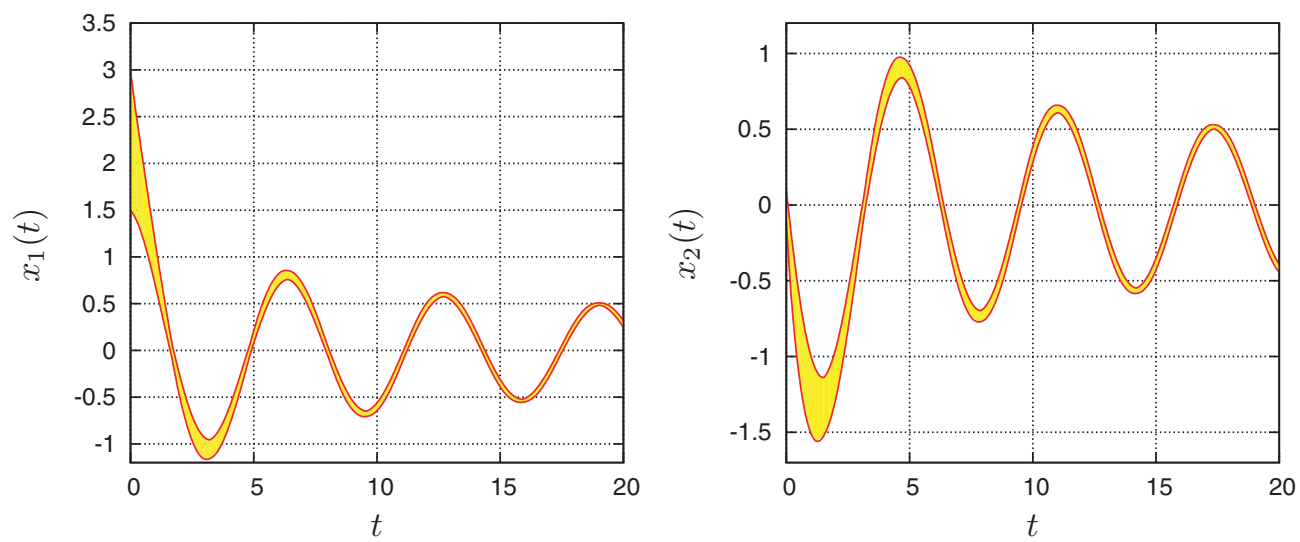

FIG. 1. Projections of the exact reachable set $X(t)$ (shaded area) on the state component $x_{1}(t)$ (left plot) and the state component $x_{2}(t)$ (right plot). The solid lines show the upper and lower bounds computed with Algorithm 1 over the time horizon [0,20].
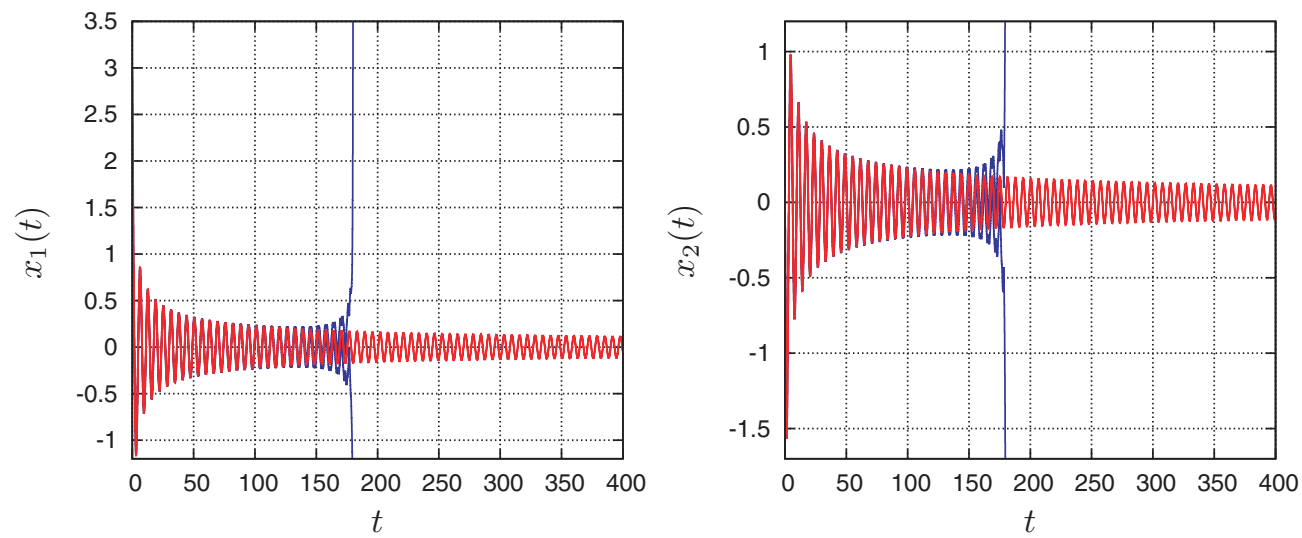

FIG. 2. Projections on the state component $x_{1}(t)$ (left plot) and the state component $x_{2}(t)$ (right plot) of the upper and lower bounds computed with Algorithm 1 (red lines) and VSPODE (blue lines) over the extended time horizon [0,400].

when propagating Taylor models with ellipsoidal remainders. In contrast, the bounds computed using VSPODE eventually explode around $t=180$, a behavior attributed to the propagation of Taylor models with interval remainder, which only enjoy linear Hausdorff convergence in the sense of Definition 2.5 (see also Remark 2).

Closely related to stability is the question of how closely the computed enclosure $\operatorname{Im}_{\mathbb{E}_{\ell}^{\text {pol }(4)} \times \mathbb{E}_{2}^{\text {ball }}}\left(Q_{x}(\cdot, P)\right)$ approximates the actual reachable set $X(t, P)$. This approximation error can be quantified as the diameter of the ellipsoidal remain$\operatorname{der} \operatorname{Im}_{\mathbb{E}_{2}^{\text {ball }}}\left(Q_{x}^{\mathrm{rem}}(\cdot, P)\right)$ with $Q_{x}^{\mathrm{rem}}(t, P) \in \mathbb{R}^{2 \times 3}$ such that $\left[Q_{x}^{\mathrm{pol}}(t, P), Q_{x}^{\mathrm{rem}}(t, P)\right]:=$ $Q_{x}(t, P)$. The left plot on Figure 3 represents the evolution of such a diameter along the time horizon for various Taylor model expansion orders $q=2, \ldots, 5$. In the case of $q=2$, Algorithm 1 stops with an error message around $t=35$, after a dramatic increase in the diameter of the ellipsoidal remainder. This is due to the fact that, in this instance, the inherent stability of the cubic oscillator system (5.1) is over-powered by the wrapping effect inherent to the ellipsoidal remainder term of the Taylor model 

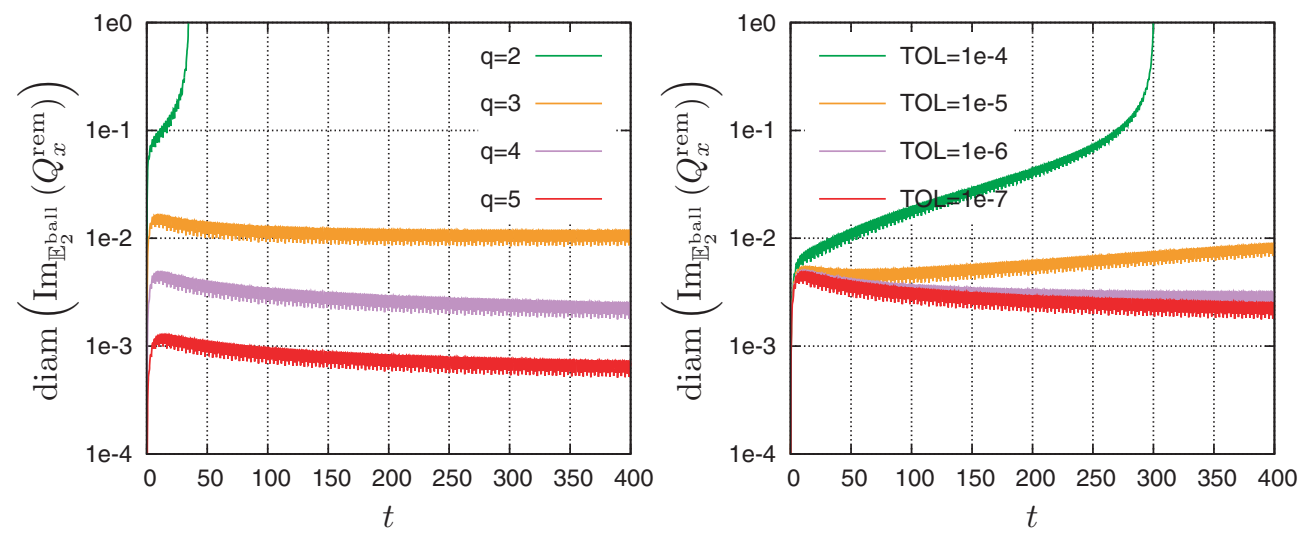

FIG. 3. Diameter of the remainder ellipsoid in the parameterization of the reachable set enclosure diam $\left(\operatorname{Im}_{\mathbb{E}_{2}^{\text {ball }}}\left(Q_{x}^{\mathrm{rem}}\right)\right)$ computed with the set-valued integrator for various Taylor model orders $q=2, \ldots, 5$ (left plot) and various tolerances $\mathrm{TOL}=10^{-4}, \ldots, 10^{-7}$ (right plot).

(for the given uncertainty set $P$ ). In contrast, the reachable set enclosures can be stabilized with higher-order expansions $q \geq 3$; that is, Algorithm 1 can in principle propagate these enclosures ad infinitum. It is also seen that the approximation error can be reduced by increasing the expansion order $q$ of the Taylor model. While this trend could be confirmed for other examples as well, the question whether or not the approximation error converges to zero in the limit remains open.

Finally, the right plot on Figure 3 represents the evolution of the diameter of the ellipsoidal remainder for various tolerance values $\mathrm{TOL}=10^{-4}, \ldots, 10^{-7}$ in the case of fourth-order Taylor models $(q=4)$. These results illustrate the effect of the parameter TOL on the integrator stability. In agreement with the asymptotic stability conditions in Definition 4.1, too large a value for TOL can indeed lead to instability of the computed enclosures and bound explosion in finite time.

6. Conclusions. This paper has presented a new discretized set-valued integration algorithm for parametric initial value problems in ODEs. This algorithm uses a predictor-validation approach to propagate generic affine set-parameterizations, whose images are guaranteed to enclose the ODE reachable tube by Theorem 3.1. We have also derived sufficient conditions in Theorem 4.2 for this algorithm to be locally asymptotically stable, in the sense that the computed reachable set enclosures are guaranteed to remain stable on infinite time horizons when applied to a dynamic system in the neighborhood of a locally asymptotically stable periodic orbit (or equilibrium point). Such stability hinges on the ability to compute quadratically Hausdorff convergent extensions in the chosen affine set-parameterization. In particular, we have described a generic way of constructing such extensions when that affine set-parameterization is invariant under affine transformation. These stability properties are illustrated for simple dynamic systems using an in-house library named CRONOS based on the library $\mathrm{MC}++$, both freely available from http://omega-icl.bitbucket.org/. Another open-source implementation is available as a subpackage in the ACADO Toolkit [12] at http://www.acadotoolkit.org. It is worth mentioning that a similar stability analysis can be conducted for continuous-time set-valued integrators, such as the ones described in [38]. In future work, implicit integration schemes will also be considered in order to improve the integrator stability, especially for stiff dynamic systems. 
Appendix A. On invariance of the cycle time T. In connection to Assumption 1, the following result establishes that the cycle time $T$ is independent of $t$ under mild conditions.

Proposition A.1. Suppose that the right-hand side function $f$ is continuously differentiable and can be rewritten in the form $f(t, x, p)=\hat{f}(\xi(t), x, p)$, where the function $\xi$ is periodic with $\xi(t+T)=\xi(t)$ for all $t$. Suppose further that there exists a parameter $p^{*}$ such that the associated limit cycle $\bar{x}\left(t, p^{*}\right)$, defined implicitly by

$$
\bar{x}\left(t, p^{*}\right):=x\left(t, \bar{x}_{0}\left(p^{*}\right), p^{*}\right) \quad \text { with } \bar{x}_{0} \text { such that } \bar{x}_{0}\left(p^{*}\right)=x\left(T, \bar{x}_{0}\left(p^{*}\right), p^{*}\right)
$$

satisfies condition (4.5), that is, is locally asymptotically stable. Then, there exists an open neighborhood $P \subseteq \mathbb{R}^{n_{p}}$ with $p^{*} \in P$ and a cycle time $T>0$ such that the limit cycles $\bar{x}(t, p)$ satisfy (4.1) for all $p \in P$. In other words, the cycle time $T$ of the limit cycles is locally independent of $p$.

Proof. The proof follows directly by applying the implicit function theorem to the nonlinear equation

$$
0=\bar{x}_{0}\left(p^{*}\right)-x\left(T, \bar{x}_{0}\left(p^{*}\right), p^{*}\right),
$$

since the associated Jacobian matrix $I-G\left(T, 0, p^{*}\right)$ is indeed invertible from the asymptotic stability condition (4.5).

Remark 5. Consider Example 6. If parameter $p_{2}$ is fixed, then the assumptions in Proposition A.1 are satisfied upon choosing $\xi(t)=\sin \left(p_{2} t\right)$. But if $p_{2}$ is allowed to vary, then the function $\xi$ depends on an unknown parameter, which is not allowed in Proposition A.1.

Appendix B. Ellipsoidal set arithmetics. The numerical computations in section 5 hinge on ellipsoidal set-parameterizations of the form $\left(\mathbb{E}_{\ell}^{\text {ball }}, \mathbb{R}^{n \times(\ell+1)}\right)$, with $\ell=n$ in order to represent ellipsoids with nonzero volume in $\mathbb{R}^{n}$ - see Example 1. Given any two set-parameterizations $\left(R_{1}, r_{1}\right),\left(R_{2}, r_{2}\right) \in \mathbb{R}^{n \times(n+1)}$, a regular addition extension $\uplus$ is given by

$$
\left(R_{1}, r_{1}\right) \uplus\left(R_{2}, r_{2}\right):=\left(\left[\frac{1}{\lambda_{1}} R_{1}^{\mathrm{T}} R_{1}+\frac{1}{\lambda_{2}} R_{2}^{\mathrm{T}} R_{2}\right]^{1 / 2}, r_{1}+r_{2}\right),
$$

where $[\cdot]^{1 / 2}$ denotes any (e.g., the symmetric) matrix square-root, and

$$
\lambda_{1}=1-\lambda_{2}=\frac{\sqrt{\operatorname{tr}\left(R_{1}^{\mathrm{T}} R_{1}\right)+\epsilon}}{\sqrt{\operatorname{tr}\left(R_{1}^{\mathrm{T}} R_{1}\right)+\epsilon}+\sqrt{\operatorname{tr}\left(R_{2}^{\mathrm{T}} R_{2}\right)+\epsilon}},
$$

with $\epsilon>0$ a small numerical regularization to prevent division by zero in degenerate cases; see, e.g., $[14,17]$ for a proof.

More specifically, the set-valued integration results in section 5 involve propagating affine set-parameterizations of the form $\left(\mathbb{E}_{\ell}^{\text {pol }(q)} \times \mathbb{E}_{n}^{\text {ball }}, \mathbb{R}^{n \times\left(\alpha_{\ell}^{(q)}+n+1\right)}\right)$ - see Example 2, namely, Taylor models with ellipsoidal remainders. Given the regular addition extension (B.1) above, and since the sum of two $q$ th-order polynomials is itself a $q$ th-order polynomial, defining a regular addition extension $\uplus$ for such parameterizations is straightforward. In turn, quadratical Hausdorff convergence of the extension (2.5) follows from Theorem 2.7. 
Acknowledgments. We are grateful to M.A. Stadtherr and Y. Lin for providing their code, VSPODE, which is used for the numerical comparison in section 5 .

\section{REFERENCES}

[1] M. Berz, From Taylor series to Taylor models, in Nonlinear Problems in Accelerator Physics, American Institute of Physics, College Park, MD, 1997, pp. 1-27.

[2] M. Berz AND G. HoffstÄtTer, Computation and application of Taylor polynomials with remainder bounds, Reliab. Comput., 4 (1998), pp. 83-97.

[3] M. Berz AND K. Makino, Performance of Taylor model methods for validated integration of $O D E s$, in Applied Parallel Computing, Lecture Notes in Comput. Sci. 3732, Springer, Berlin, 2006, pp. 65-74.

[4] F. Blanchini and S. Miani, Set-Theoretic Methods in Control, Birkhäuser, Boston, 2008.

[5] A. Bompadre, A. Mitsos, And B. Chachuat, Convergence analysis of Taylor and McCormick-Taylor models, J. Global Optim., 57 (2013), pp. 75-114.

[6] B. Chachuat, A.B. Singer, And P.I. Barton, Global methods for dynamic optimization and mixed-integer dynamic optimization, Ind. Eng. Chem. Res., 45 (2006), pp. 8373-8392.

[7] B. Chachuat and M.E. Villanueva, Bounding the solutions of parametric ODEs: When Taylor models meet differential inequalities, in Proceedings of the 22nd European Symposium on Computer Aided Process Engineering, Vol. 30, I.D.L. Bogle and M. Fairweather, eds., 2012, pp. 1307-1311.

[8] G.F. CoRliss AND R. Rinm, Validating an a priori enclosure using high-order Taylor series, in Proceedings of the International Symposium on Scientific Computing, Computer Arithmetic and Validated Numerics-SCAN'95, G. Alefeld, A. Frommer, and B. Lang, eds., Akademie Verlag, Berlin, 1996, pp. 228-238.

[9] A. Griewank, Evaluating Derivatives: Principles and Techniques of Algorithmic Differentiation, Frontiers Appl. Math. 19, SIAM, Philadelphia, 2000.

[10] E. Hairer and G. Wanner, Solving Ordinary Differential Equations II-Stiff and DifferentialAlgebraic Problems, Springer, Berlin, 1991.

[11] A.C. Hindmarsh, P.N. Brown, K.E. Grant, S.L. Lee, R. Serban, D.E. Shumaker, and C.S. WOODWARD, SUNDIALS: Suite of nonlinear and differential/algebraic equation solvers, ACM Trans. Math. Software, 31 (2005), pp. 363-396.

[12] B. Houska, H.J. Ferreau, And M. Diehl, ACADO Toolkit-An open source framework for automatic control and dynamic optimization, Optimal Control Appl. Methods, 32 (2011), pp. 298-312.

[13] B. Houska, Robust Optimization of Dynamic Systems, Ph.D. thesis, Katholieke Universiteit Leuven, 2011.

[14] B. Houska, F. Logist, J. Van Impe, and M. Diehl, Robust optimization of nonlinear dynamic systems with application to a jacketed tubular reactor, J. Process Control, 22 (2012), pp. $1152-1160$.

[15] B. Houska AND B. СнасHUAт, Branch-and-lift algorithm for deterministic global optimization in nonlinear optimal control, J. Optim. Theory Appl., 162 (2014), pp. 208-248

[16] B. Houska, M.E. Villanueva, and B. Chachuat, A validated integration algorithm for nonlinear ODEs using Taylor models and ellipsoidal calculus, in Proceedings of the 2013 IEEE 52nd Annual Conference on Decision and Control (CDC), pp. 484-489.

[17] A.B. Kurzhanski And P. Valyi, Ellipsoidal Calculus for Estimation and Control, Birkhäuser, Boston, 1997.

[18] Y. Lin And M.A. Stadtherr, Validated solutions of initial value problems for parametric ODEs, Appl. Numer. Math., 57 (2007), pp. 1145-1162.

[19] Y. Lin AND M.A. STADTHERR, Deterministic global optimization of nonlinear dynamic systems, AIChE J., 53 (2007), pp. 866-875.

[20] R.J. Lohner, Computation of guaranteed enclosures for the solutions of ordinary initial and boundary value problems, in Computational Ordinary Differential Equations, J.R. Cash and I. Gladwell, eds., Clarendon Press, Oxford, 1992, pp. 425-436.

[21] G.P. McCoRmick, Computability of global solutions to factorable nonconvex programs: Part I - Convex underestimating problems, Math. Program., 10 (1976), pp. 147-175.

[22] A. Mitsos, B. Chachuat, and P.I. Barton, McCormick based relaxations of algorithms, SIAM J. Optim., 20 (2009), pp. 573-601.

[23] R.E. Moore and F. Bierbaum, Methods and Applications of Interval Analysis, SIAM, Philadelphia, 1979. 
[24] R.E. Moore, R.B. Kearfott, and M.J. Cloud, Introduction to Interval Analysis, SIAM, Philadelphia, 2009.

[25] U. Naumann, The Art of Differentiating Computer Programs: An Introduction to Algorithmic Differentiation, SIAM, Philadelphia, 2009.

[26] N.S. Nedialkov, K.R. Jackson, and G.F. Corliss, Validated solutions of initial value problems for ordinary differential equations, Appl. Math. Comput., 105 (1999), pp. 21-68.

[27] M. Neher, K.R. JaCkson, And N.S. Nedialkov, On Taylor model based integration of ODEs, SIAM J. Numer. Anal., 45 (2007), pp. 236-262.

[28] A. Neumaier, Taylor forms-Use and limits, Reliab. Comput., 9 (2002), pp. 43-79.

[29] I. PAPAMichail AND C.S. AdJiman, A rigorous global optimization algorithm for problems with ordinary differential equations, J. Global Optim., 24 (2002), pp. 1-33.

[30] J.B. Rawlings And D.Q. Mayne, Model Predictive Control: Theory and Design, Nob Hill, Madison, WI, 2009.

[31] A.M. Sahlodin And B. Chachuat, Convex/concave relaxations of parametric ODEs using Taylor models, Comput. Chem. Eng., 35 (2011), pp. 844-857.

[32] A.M. Sahlodin And B. Chachuat, Discretize-then-relax approach for convex/concave relaxations of the solutions of parametric ODEs, Appl. Numer. Math., 61 (2011), pp. 803-820.

[33] J.K. Scott, B. Chachuat, ANd P.I. Barton, Nonlinear convex and concave relaxations for the solutions of parametric ODEs, Optimal Control Appl. Methods, 34 (2013), pp. 145-163.

[34] J.K. SCOTT AND P.I. BARTON, Improved relaxations for the parametric solutions of ODEs using differential inequalities, J. Global Optim., 57 (2013), pp. 143-176.

[35] A.B. Singer, Global Dynamic Optimization, Ph.D. thesis, Massachusetts Institute of Technology, Cambridge, MA, 2004.

[36] A.B. Singer And P.I. Barton, Bounding the solutions of parameter dependent nonlinear ordinary differential equations, SIAM J. Sci. Comput., 27 (2006), pp. 2167-2182.

[37] G. Teschl, Ordinary Differential Equations and Dynamical Systems, Grad. Stud. Math. 140, AMS, Providence, RI, 2012.

[38] M.E. Villanueva, B. Houska, and B. Chachuat, Unified framework for the propagation of continuous-time enclosures for parametric nonlinear ODEs, J. Global Optim., 62 (2015), pp. 575-613.

[39] W. WaLter, Differential and Integral Inequalities, Springer, Berlin, 1970. 